\title{
A Compact Manufacturable 76-77-GHz Radar Module for Commercial ACC Applications
}

\author{
I. Gresham, Member, IEEE, N. Jain, Member, IEEE, T. Budka, Member, IEEE, A. Alexanian, Member, IEEE, \\ N. Kinayman, Member, IEEE, B. Ziegner, S. Brown, and P. Staecker, Fellow, IEEE
}

\begin{abstract}
The design and measured results of a single-substrate transceiver module suitable for 76-77-GHz pulsed-Doppler radar applications are presented. Emphasis on ease of manufacture and cost reduction of commercial millimeter-wave systems is employed throughout as a design parameter. The importance of using predictive modeling techniques in understanding the robustness of the circuit design is stressed. Manufacturing techniques that conform to standard high-volume assembly constraints have been used. The packaged transceiver module, including three waveguide ports and intermediate-frequency output, measures 20 $\mathrm{mm} \times 22 \mathrm{~mm} \times 8 \mathrm{~mm}$. The circuit is implemented using discrete GaAs/AIGaAs pseudomorphic high electron mobility transistors (pHEMTs), GaAs Schottky diodes, and varactor diodes, as well as GaAs p-i-n and pHEMT monolithic microwave integrated circuits mounted on a low-cost $127-\mu \mathrm{m}$-thick glass substrate. A novel microstrip-to-waveguide transition is described to transform the planar microstrip signal into the waveguide launch. The module is integrated with a quasi-optical antenna. The measured performance of both the component parts and the complete radar transceiver module is described.
\end{abstract}

Index Terms-Circuit functions, consumer electronics, Doppler radar, millimeter-wave radar, waveguide transitions.

\section{INTRODUCTION}

$\mathbf{T}$ HE FIELD of radar for automotive applications and other millimeter-wave sensors is currently attracting great interest and investment [1], although work in the field has been going onfor many years [2]. The techniques required to provide the desired level of system performance are not new and have formed the basis of many military applications in radar and sensing for more than 30 years [3]. The stumbling block that has prevented the implementation of these sophisticated systems in commercial arenas such as the automotive industry is the disparity between the traditional cost of a millimeter-wave radar system and the price that a consumer is willing to pay. The focus to date, therefore, has been hardware demonstration that fulfills the required functionality [4], [5]. Nevertheless, several operating autonomous cruise control (ACC) systems have been developed and demonstrated - often in conjunction with automotive manufacturers - and a few are now in production and commercially available, although at a premium price [1], [6]-[11].

Manuscript received February 21, 2000; revised August 25, 2000.

I. Gresham, N. Kinayman, B. Ziegner, and S. Brown are with M/A-COM, Lowell, MA 01853 USA (e-mail: igresham@ieee.org).

$\mathrm{N}$. Jain is with the Indian Institute of Science, Bangalore, India.

T. Budka and A. Alexanian are with RF Micro Devices, Billerica, MA 01821

USA (e-mail: budka@ieee.org).

P. Staecker, retired, was with M/A-COM, Lowell, MA 01853 USA. He is now

at 167 Cedar Street, Lexington, MA 02421 USA.

Publisher Item Identifier S 0018-9480(01)01549-6.
However, the radar systems currently available are unlikely to meet the projected cost profile required for commercial deployment. Since the projected demand for these systems is millions of units per year, the problem of low-cost high-yield manufacturing and test is a significant challenge. Recent advances in simulation capability and resources, semiconductor manufacturing [12]-[14], and assembly techniques have focused on solving this problem through the development of monolithic microwave integrated circuit (MMIC) building blocks [15]-[25]. However, the problem of device integration, their packaging, and providing other mechanical and electrical interfaces remains a technically challenging and potentially costly issue that has not been adequately addressed [26], [27].

The intent of this paper is to address these issues at a fundamental level. The module design is driven equally by considerations of ease — of manufacture and cost —and functional considerations. This philosophy necessitates accurate modeling and characterization of the circuit, including yield analysis under process drift, manufacturing tolerance, and statistical variation. Though a complete millimeter-wave transceiver module suitable for use in a commercial ACC radar system is described, the philosophy underlying the design holds for other applications as well.

Section II of this paper describes some of the system constraints of ACC radar modules and their implication for mechanical and electrical design of the transceiver. This information is used to explain the choice of the selected circuit configuration. The design and performance of the individual components is covered in Section III. The transceiver module is integrated with an antenna from a commercial ACC system, and the corresponding results are reported in Section IV. This paper concludes with a summary in Section V.

\section{ACC RADAR SYSTEM CONSTRAINTS}

The millimeter-wave components of a radar transceiver can be broadly classified in two parts: 1) the circuit functions - signal generation, conversion, and routing — and 2) the antenna. The challenge of making antennas that are compatible with the requirements of the automotive industry such as low cost, compact size, and possibly either electrically or mechanically steered plus harsh environmental considerations, is also attracting much attention [28], [29].

Fig. 1 shows a simplified block diagram of the complete radar system that illustrates the interface between the millimeter-wave module and the other required circuitry. Although the system considered in this case is that of pulsed-Doppler radar, many 


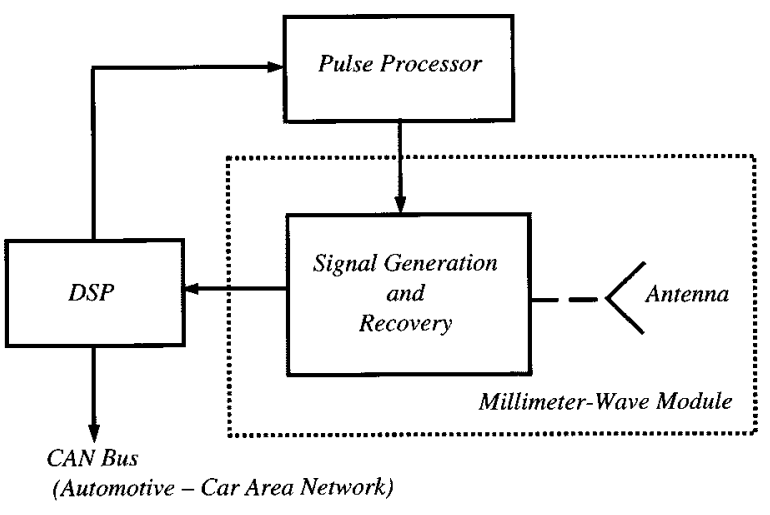

Fig. 1. Simplified block diagram of a pulsed-Doppler radar system.

of the technical challenges and certainly all of the manufacturing challenges are identical for other millimeter-wave radar systems. The relative advantages and disadvantages of the different system architectures are not within the scope of this paper and have been well covered elsewhere [3].

The millimeter-wave module consists of a signal generation and recovery stage that interfaces with the antenna. More than one antenna may be used in some applications, but the principle remains the same. The interfaces between the millimeter-wave module and the control and digital-signal-processing (DSP) circuitry are at relatively low intermediate frequency (IF) or baseband frequencies. The choice of configuration of the signal generation and recovery circuitry can be made by considering the following issues.

\section{A. Performance Issues of Using MMICs as Frequency Sources and the Advantages of Low-Frequency Signal Generation}

Traditionally, millimeter-wave signal generation has relied on circuits using transferred electron devices (TEDs) and other two-terminal devices; these form the basis of some of the ACC systems available today. Although TEDs can generate significant amounts of power, and their low-flicker noise characteristic results in low-phase-noise sources, the assembly and alignment cost of cavity-based oscillators tends to make them expensive. Furthermore, their poor conversion efficiency $(\sim 2 \%)$ and physical device structure makes the implementation of planar TED-based oscillators a challenge for electrical and thermal reasons [30], [31]. Although several authors have attempted to minimize the complexity and, therefore, the part count of the millimeter-wave circuitry by fundamental signal generation at $76 \mathrm{GHz}$ using MMICs [32]-[38], there are compelling performance and manufacturing reasons to generate power with oscillators operating at lower frequencies. This necessitates some form of upconversion to realize the $W$-band signal.

From a performance consideration, it is easier to generate low-phase-noise oscillators at lower frequencies [39], [40]. Phase noise is an important parameter in both frequency-modulated continuous-wave (FMCW) and pulsed radar systems-in the former because of the homodyne detection architecture and in the latter due to the requirements on pulse-to-pulse stability [3], [41]. Reducing the phase noise of the $W$-band source using high $Q$ structures such as a dielectric resonator is very difficult [42]. Phase-locking the source to a stable low-frequency oscillator can provide noise reduction, though at a considerable expense [43], [44].

In addition to the phase-noise issues of MMIC $W$-band oscillators, the temperature stability of the oscillators is also relatively poor [45] and requires compensation. This has performance implications for both pulsed and FMCW systems in addition to the legal requirement to operate within a defined band of the frequency spectrum. The ability of FMCW radar systems to identify range and discriminate between targets is a direct function of the tuning linearity of the oscillator, which varies as a function of temperature. The temperature stability of an oscillator can be greatly improved by using a lower frequency source combined with a frequency multiplier [46]. The buffering that this technique provides between the oscillator and the antenna also helps to reduce frequency pulling. As the antenna beam is scanned, changes in the load impedance due to the proximity of other vehicles causes frequency pulling of the source. Although the use of a buffer amplifier at $W$-band to increase the output power of the oscillator helps in reducing this effect, the isolation it provides may be less than that available from a frequency multiplier and amplifier. Additional considerations are the relative availability and cost of these devices. In addition, the increased resonator $Q$ at lower frequencies also makes the oscillator less susceptible to frequency pulling.

The advantages for manufacturing in producing lower frequency signal generation include greater tolerance of manufacturing error, lower cost materials and semiconductors (including discrete devices), and the large experience base of high-volume assembly techniques at these frequencies.

\section{B. Optimum System Architecture for Cost and Ease-of-Manufacturing Reasons}

The precise system architecture needed to address a specific performance requirement is flexible. Given this fact, the subsequent choice of the circuitry when designing for commercial applications should be made predominantly for material cost and ease-of-manufacturing reasons.

The impetus to use MMICs in automotive applications arises from seeing the effect that high-volume markets have had upon the associated pricing on GaAs technologies at lower frequencies due to the expansion of the wireless market [47]. The presence of a high-volume consumer application coupled with competing technologies such as $\mathrm{Si}$ or SiGe forced the cost reduction of GaAs IC-based products. This was achieved by a combination of increasing yield, increasing wafer sizes to first $100 \mathrm{~mm}$ and now $150 \mathrm{~mm}$, and introducing automated test techniques that were already well established for lower frequency products. The situation is quite different for the millimeter-wave arena, and although cost reduction is predicted [48], the rate of progress is not compatible with that desired by the automotive industry [49]. Aside from the high volume required to reduce pricing, there are several technical reasons that must be considered in selecting the appropriate circuit configuration and technology.

Process requirements for millimeter-wave devices are more exacting than they are for wireless applications. Raffaelli reviewed the available technologies suitable for automotive radar applications and concluded that there were two principal options, both of which have their drawbacks [50]. Metal-semicon- 
ductor field-effect transistor (MESFET) devices with a $0.25-\mu \mathrm{m}$ gate length cannot be effectively used above $38 \mathrm{GHz}$, while pseudomorphic high electron mobility transistor (pHEMT) devices capable of operating at $77 \mathrm{GHz}$ have two issues: they require a short gate length $(<0.2 \mu \mathrm{m})$ that cannot be defined optically and are principally InP based. InP devices potentially offer better performance for amplifiers at $W$-band due to their increased mobility when compared with GaAs. Available devices are still largely from 3 -in processes, although the move to $100-\mathrm{mm}$ wafers is slowly happening. Yield is also a cost driver for these devices, with a high yield design at $W$-band defined as being $>50 \%$ [51]. The first of these issues has been recognized as a cost driver by Wethof et al. [52], who have demonstrated a $W$-band amplifier based on $0.25-\mu \mathrm{m}$ GaAs HEMTs, although the 1-dB compression point of the amplifier was only $+9 \mathrm{dBm}$. A study by Morenc [53], similar to that of Raffaelli, concluded that the way to realize the circuitry required for automotive radar at the lowest cost was to generate the signal at as low a frequency as possible-using discrete devices where appropriate-and multiply the signal up to $W$-band.

The second area where $W$-band monolithic and planar circuits cannot utilize the advances made by the burgeoning wireless market is packaging and interconnects. This problem-at least of the same importance as that of device selection and circuit configuration - has been relatively neglected. Regardless of the devices and circuit used to generate and control the 77-GHz signal, the problem of connecting devices with each other and providing electrical and mechanical interfaces to the antenna and control circuitry is a significant challenge. This was recognized by Takahashi et al. [54], who demonstrated a 30-GHz receiver front end by mounting active devices on a low-loss benzocyclobutene (BCB) dielectric substrate on an Si carrier. All of the interconnects were made using this dielectric substrate. Discrete devices rather than MMICs were used to minimize the area of expensive heterostructure substrates. The importance of small chip size, low-cost packaging, and interconnects for automotive radar has more recently been stressed by Kondoh et al. [26], who mounted an MMIC chip-set on an "interconnection sheet" comprising of a dielectric substrate that also formed the reverse side of a planar antenna structure.

We can, therefore, draw some broad conclusions about the way that the millimeter-wave module should be realized. Using discrete devices where possible should minimize the area of the semiconductors; circuit functionality should be realized at a lower frequency where practical; and the selected medium should be compatible with standard manufacturing techniques and procedures. Increased signal loss at high frequency plus the increased difficulty and cost of manufacture requires that, ideally, the millimeter-wave signal be generated as close to the antenna as possible and that the receive signal be downconverted and recovered in a similar fashion. Lastly, each element in the circuit chain should be tolerant of the effects of typical manufacturing tolerance and process variation.

\section{Design And Performance of Module Components}

This paper uses the principles outlined above to design a single low-cost millimeter-wave module that is entirely com-

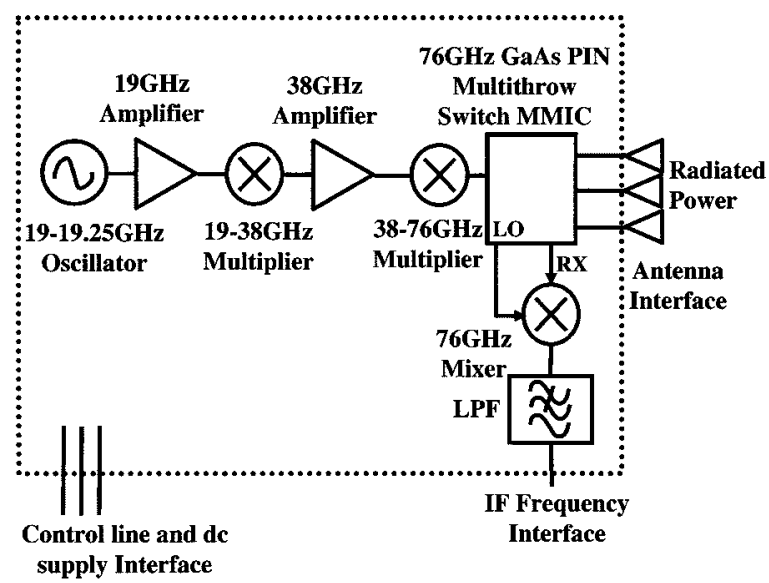

Fig. 2. Circuitry within the signal generation and recovery module showing the required interfaces.

patible with standard high-volume manufacturing practice. To minimize the area of expensive semiconductor material, discrete devices are used where possible. These devices are mounted in both a conventional upright fashion and flip-chip where appropriate. The selected circuit configuration is illustrated in Fig. 2 and emphasizes the practice of realizing the source at a relatively low frequency and multiplying to generate the $W$-band signal. The entire circuit is contained within a low-cost package whose interfaces with the other components of the radar system consist of dc supply lines, the IF signal output, and three waveguide ports that feed the antenna. Following the principle that the millimeter-wave signal should be generated and the received signal recovered as close to the antenna as possible, the module is designed to mount directly behind the radio-frequency (RF) ground plane of the antenna, thus minimizing the length of the waveguide feeds. A schematic diagram indicating the mounting method is shown in Fig. 3 [55]. The drop-in interface between the millimeter-wave module and the antenna allows the module to be independently tested and its performance characterized. The novel package technique described later in Section III allows for easy interchange and replacement of the module during production testing. This combination of low material cost, compliance to standard manufacturing techniques, and ease of integration and test describes a design approach that results in potentially low-cost products.

\section{A. Overview-System and Circuit Architecture}

The ACC system uses a switched three-beam pulsed-Doppler radar operating at 76-77 GHz. Fig. 2 illustrates the module block diagram employed. The output from a voltage controlled dielectric resonator oscillator (DRO) at $19 \mathrm{GHz}$ is amplified and multiplied to $38 \mathrm{GHz}$. The DRO, amplifier, and multiplier are based on upright bonded discrete pHEMTs and flip-chip mounted varactor diodes. The $38 \mathrm{GHz}$ signal is then further multiplied and amplified to provide an output signal at $76 \mathrm{GHz}$. In this example, the second multiplier stage was realized using a commercially available MMIC [15] for reasons of expediency in proof-of-concept. There are further cost advantages to be gained in replacing this MMIC with a $38-\mathrm{GHz}$ amplifier and a second passive multiplication stage. 


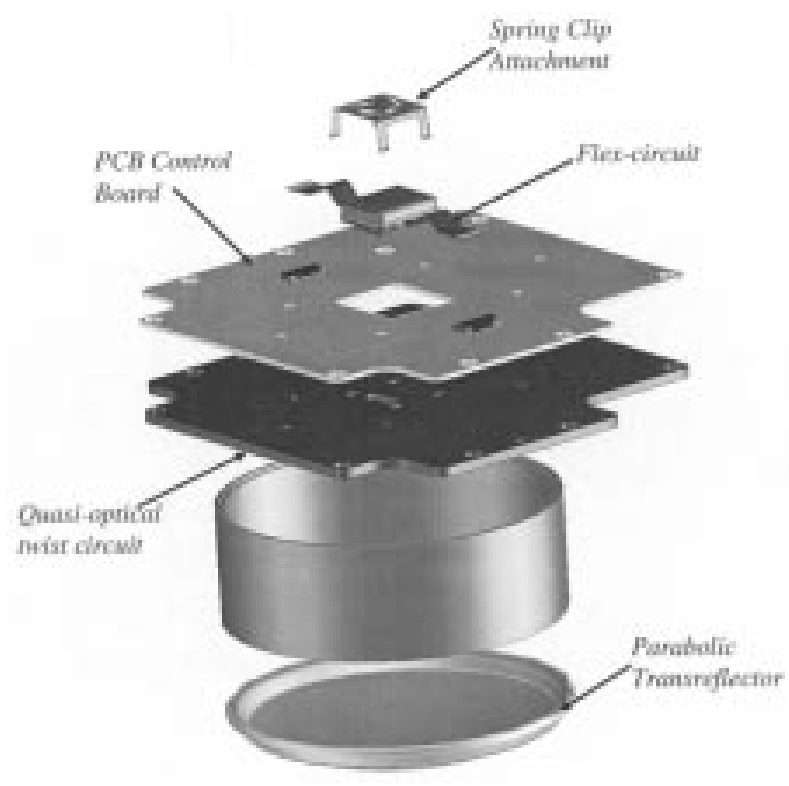

Fig. 3. Exploded diagram illustrating the module/antenna interface and assembly. The millimeter-wave module is mounted as a drop-in component on the radar control board.

When the module is in transmit mode (TX), the $76-\mathrm{GHz}$ signal is directed via the GaAs p-i-n diode switch MMIC to one of three antenna feeds providing azimuth object detection [56]. In receive mode ( $\mathrm{RX})$, the DRO is electronically tuned to an appropriate IF offset and the multiplied output switched to the local oscillator (LO) arm of the mixer. The return signal received by the antenna is then heterodyne mixed with the LO and the IF recovered.

The radar transceiver is realized by mounting discrete active devices and MMICs onto a glass substrate. Semiconductor devices are mounted by flip-chip attach for the varactor and Schottky diodes, and with normal and compensated wire-bond interconnects [57] for the pHEMTs and MMICs. The glass substrate is made using M/A-COM's proprietary passive glass microwave integrated circuit (GMIC) process [58], [59]. Silicon and glass are combined to create a low-cost circuit medium that has attractive properties for millimeter-wave design. Fig. 4 illustrates a cross-section of a typical GMIC circuit with some representative available features.

The monolithic nature of the technology allows for automated batch processing with the ability to integrate photolithography defined passive components-spiral inductors, metal-insulator-metal and interdigital capacitors, and thin-film resistors-with external active devices. In addition, the islands of Si embedded in the glass substrate provide both RF and dc ground returns as well as thermal heatsinks for the external devices and ICs. The glass provides electrical isolation, mechanical support, low dielectric constant $\left(\varepsilon_{r}=4.0\right)$, and low loss tangent $(\tan \delta=0.002$ at $10 \mathrm{GHz})$. The rigid substrate and the relatively thick Au metallization $(\sim 4 \mu \mathrm{m})$ are also ideal for die attach and wire bonds.

In this instance, the substrate contains low-loss transmission lines and biasing networks as well as provides a low-loss microstrip to waveguide interface by means of a special mode transducer and launch network described in Section III. Mi-

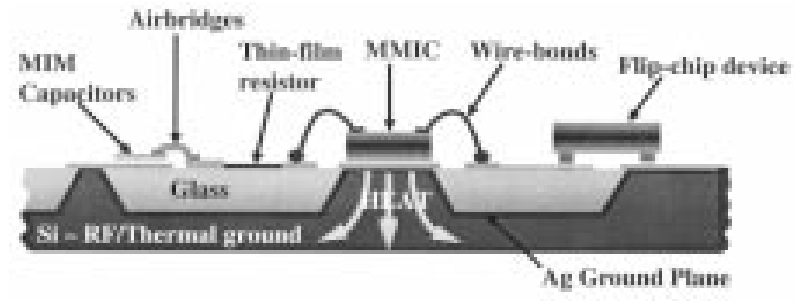

Fig. 4. Cross-section of GMIC showing available passive features.

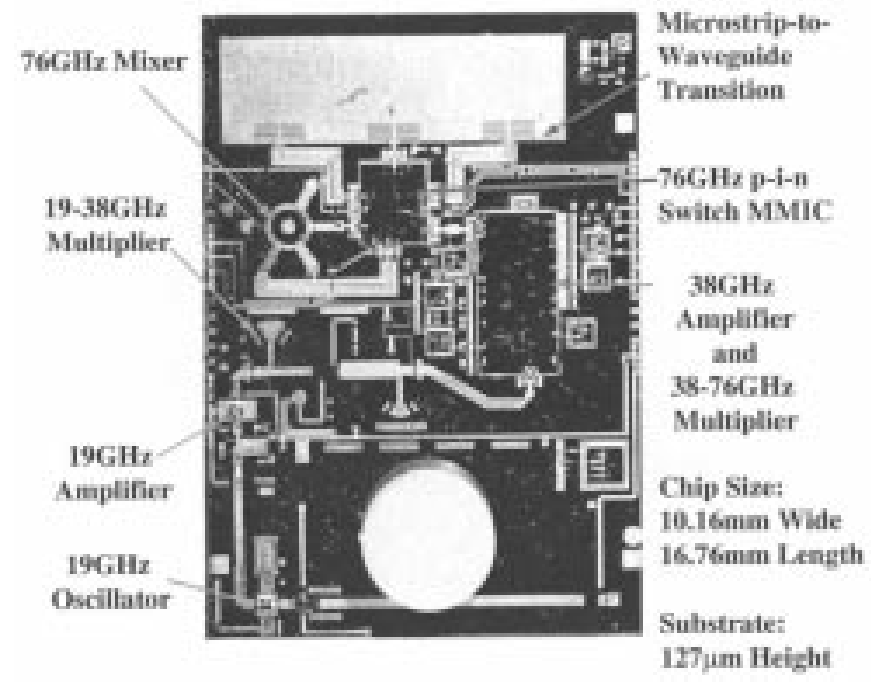

Fig. 5. The complete 76-77-GHz transceiver module as realized using a glass (GMIC) substrate.

crostrip, coplanar waveguide (CPW), lumped and distributed circuit elements, and isolating RF ground shields can and have been utilized wherever appropriate for the circuit design. All wire-bond interconnects are designed to be implemented with standard $25-\mu \mathrm{m}$ diameter ball-bonds. A photograph of the complete transceiver module is shown in Fig. 5. The substrate measures $10.16 \mathrm{~mm}$ wide by $16.76 \mathrm{~mm}$ long.

The glass substrate is mounted on a metal header that performs two functions. First, the header provides an RF ground plane and thermal heatsink for the devices mounted on the top surface of the substrate. Secondly, to facilitate assembly, handling, and test, the millimeter-wave module is required to be a self-contained unit. One of the key challenges in designing a drop-in module is how to devise the millimeter-wave connection at the module and antenna interface such that it meets both functional and assembly requirements. The circuitry is assembled on a planar substrate, yet a robust high-frequency interface between the circuit and the antenna is required. This can be accomplished with a microstrip-to-waveguide transition, as shown in Fig. 6.

The header thus forms the package base and also the mounting surface for the three waveguide feeds. The millimeter-wave interface between the transceiver module and the waveguide feeds is a tapered waveguide channel fed from a patented microstrip-to-waveguide mode transition on the front side of the glass substrate [60]. The waveguide feeds directly illuminate the antenna by mounting the module on the reverse side of the antenna ground plane. The feeds are captured in a tapered slot 


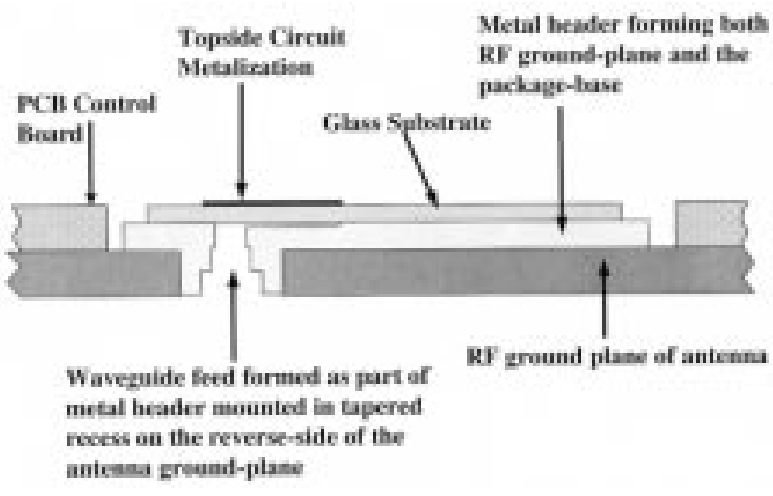

Fig. 6. Schematic cross-section through the glass substrate and the metal header showing how the drop-in package realizes the millimeter-wave waveguide interface with the antenna (drawing not to scale).

in the mounting plate by virtue of an interference fit. Other self-locating features allow rapid and repeatable installation and replacement of the module. These locators position the waveguide ports for proper antenna illumination. Careful attention to tolerance and plate thickness ensures that the antenna and the module share a continuous RF ground plane.

\section{B. Module Package}

1) Package Features and Interface Description: The millimeter-wave module requires a package that provides a low-cost rugged enclosure for environmental protection, has efficient waveguide ports for antenna illumination, and has a simple yet repeatable installation technique into the radar assembly to facilitate test and handling. Metal injection molding (MIM) technology was chosen for the fabrication of the package base and cover to satisfy the requirements for precision details and low cost in production volumes. MIM technology is particularly attractive for small metal parts that have fine details or complex geometry. The millimeter-wave module is then installed into the radar assembly or into a test fixture and retained in position using a spring clip and two flex cable connectors, as shown in Fig. 7 [55]. The spring clip allows for repeatable insertion and removal of the module without the need for solder attachment. The flexible cables insert into zero-insertion-force connectors. These cables provide both the $\mathrm{dc}$ and control lines for the module circuitry but also feed the recovered IF signal to the DSP.

2) Package Assembly Details: The glass substrate is mounted on the package base using a low modulus epoxy to compensate for the differing thermal coefficients of expansion between the glass substrate and the metal composition of the package. The dc and IF input and output lines are connected to the flex-circuit interconnect medium using standard wire-bond methods. The MIM cover includes a shield septum that defines the DRO cavity. These features are shown in Figs. 8 and 9. The cover is epoxy-sealed to the package base. The spring clip provides the pressure needed $(\sim 0.4 \mathrm{mPa})$ to seat the package in the antenna waveguide port properly as well as to ensure a consistent thermal contact to the mounting plate supporting the radar assembly. The four latching fingers of the installed spring clip securely grip the support plate, and removal is achieved by squeezing the spring clip to release these latching

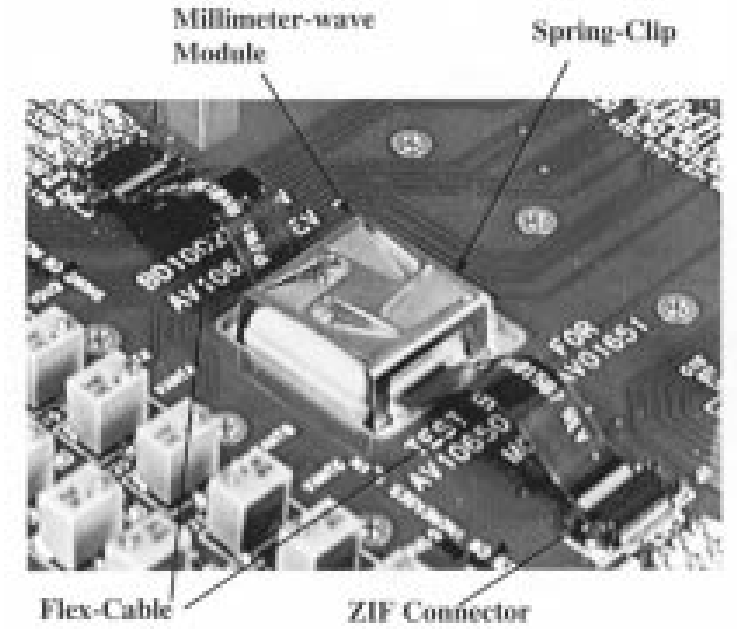

Fig. 7. Photograph of the millimeter-wave module mounted on a test board showing mounting technique with spring clip and flex cables.

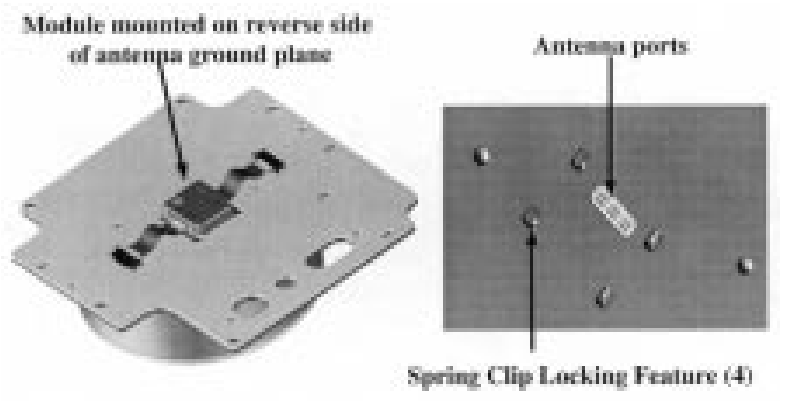

Fig. 8. Drawing of the interface between the module waveguide ports and the antenna RF ground plane as seen from the antenna.

fingers. A photograph of the module with the cover removed is shown in Fig. 10 and illustrates the interconnection between the flex-ribbon connector and the glass circuit.

\section{Module Circuit Components-Specifications, Design, and Measured Performance}

For brevity, the following details regarding the design, modeling, and measured performance of the individual circuit components are restricted to the more salient points and a summary of the measured performance. Comments regarding manufacturing issues and relevant references are given where appropriate.

The target specification for each of the components was necessarily derived by consideration of the system-level specification. However, the specified operating bounds of each of these component level circuits also need to compensate for the variation of the other circuits in the module. For example, the specification for input and output return loss, and conversion loss of the 19-38 GHz passive multiplier, need to be considered as a function of how the available input power varies as a function of manufacturing tolerance and process variation, plus dynamic considerations like ambient temperature. To ensure a robust design that not only meets performance specifications but also results in a high manufacturing yield $(>90 \%)$, it is necessary to perform either Monte Carlo or sensitivity analysis-where possible - on each of the circuit designs to ensure that these performance bounds are met. 


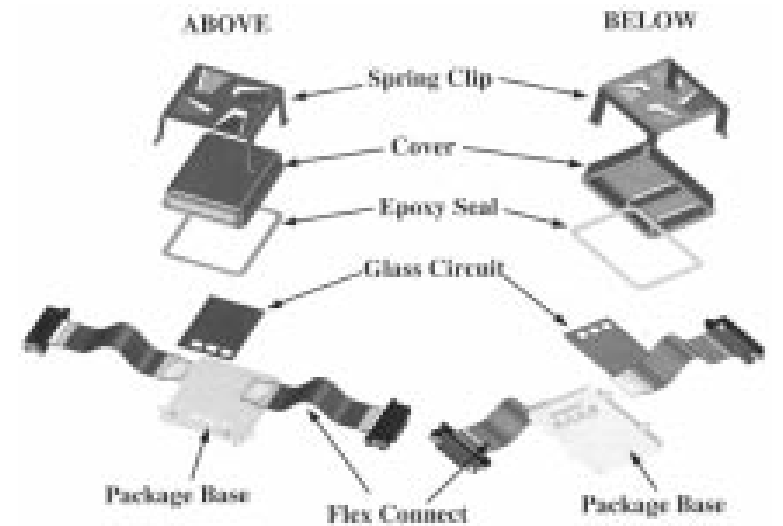

Fig. 9. Exploded drawing illustrating the assembly of the package to form the complete millimeter-wave module (from above and below).

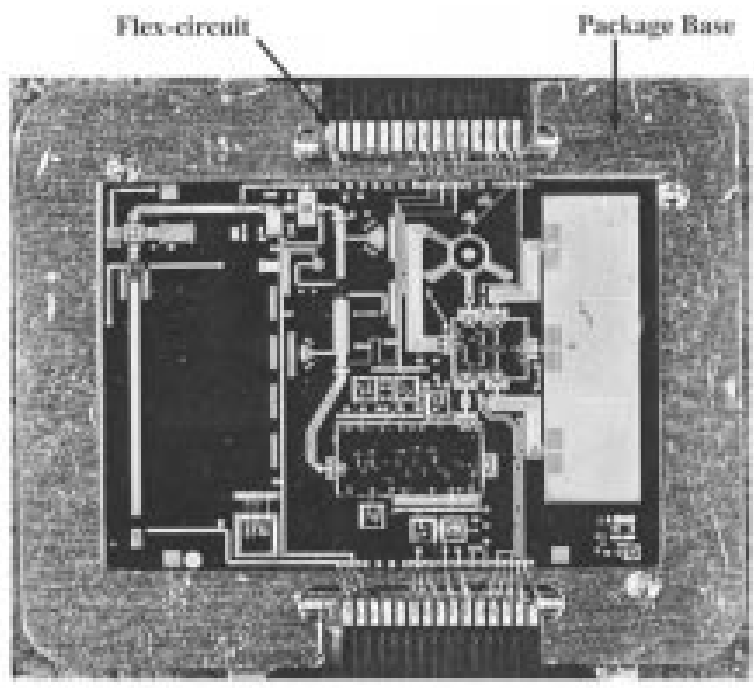

Fig. 10. Photograph of the glass circuit mounted on the module base illustrating the flex-circuit interconnections.

1) 19-GHz DRO and Buffer Amplifier: The oscillator uses the series-feedback or reflection-mode configuration. The dielectric resonator is coupled to a $50-\Omega$ transmission line connected to the gate terminal. This arrangement helps to isolate the oscillator frequency further from frequency-pulling effects caused by changes in the antenna load impedance. The frequency of the oscillator is varied by adjusting the reverse-bias voltage of a varactor diode, which is used as an impedancetransformation or phase-shift element between the resonator and the pHEMT [61]-[63]. This configuration has the following advantages.

1) The position of the diode in the transmission line can be optimized to maximize the tuning range.

2) The problem of modeling the oscillator is greatly simplified.

3) The tolerance to manufacturing error is reduced.

4) The manufacturing process itself is extremely simple.

For these reasons, this technique is applicable for high-volume applications. The active device used for both the 19-GHz oscillator and the single-ended buffer amplifier is a discrete GaAs
TABLE I

SuMmary OF THE Key OSCILlator Characteristics AND SimUlation INFORMATION

\begin{tabular}{|c|c|}
\hline Circuit & $\begin{array}{l}\text { - Reflection-mode DRO based on self-biased pHEMT } \\
\text { (upright mounted) }\end{array}$ \\
\hline \multirow{4}{*}{ Configuration } & - GaAs hyperabrupt varactor diode (flip-chip mounted) \\
\hline & - Single-ended buffer amplifier (self-biased) \\
\hline & $\begin{array}{l}\text { - Nonlinear pHEMT model extracted from I-V and S- } \\
\text { parameter measurements }\end{array}$ \\
\hline & $\begin{array}{l}\text { - Diode model extracted from S-parameter and dc } \\
\text { measurements (junction) plus full-wave } \\
\text { electromagnetic (EM) analysis for package }\end{array}$ \\
\hline \multirow[t]{3}{*}{ Models } & $\begin{array}{l}\text { - Resonator model derived from numerical analysis and } \\
\text { compared with full-wave EM model. Curve-fitting } \\
\text { used to optimize fit [62-66] }\end{array}$ \\
\hline & $\begin{array}{l}\text {-Full-wave EM analysis of circuit structures and } \\
\text { discontinuities }\end{array}$ \\
\hline & $\begin{array}{l}\text { - Small-signal analysis used for insight into operating } \\
\text { characteristics }\end{array}$ \\
\hline \multirow[t]{2}{*}{ Techniques } & $\begin{array}{l}\text {-Harmonic-balance analysis to predict RF power/bias } \\
\text { dependency/output spectrum }\end{array}$ \\
\hline & $\begin{array}{l}\text { - Transient analysis to model frequency switching-speed } \\
\text { under pulsed conditions }\end{array}$ \\
\hline \multirow[t]{2}{*}{$\begin{array}{l}\text { Yield } \\
\text { Analysis }\end{array}$} & $\begin{array}{l}\text { - Sensitivity analysis of oscillator performance as a } \\
\text { function of process tolerance (device/circuit) and } \\
\text { manufacturing error (puck placement) }\end{array}$ \\
\hline & $\begin{array}{l}\text {-Monte-Carlo analysis of amplifier (gain/ } \mathbf{S}_{11} \text { and } \\
\mathrm{S}_{22} \text { /output power) }\end{array}$ \\
\hline
\end{tabular}

pHEMT. All wire-bonds are standard $25-\mu \mathrm{m}$ ball-bonds as typically available from a high-speed automated bonding machine. The key oscillator characteristics, with details on circuit models and modeling techniques used in the design, are summarized in Table I.

The purpose of the 19-GHz buffer amplifier is twofold: 1) to provide a better defined output impedance for the oscillator-close to $50 \Omega$-rather than the sensitive input match of the frequency doubler and 2) to drive the frequency doubler with a constant RF signal level over temperature to minimize power variation within the module. The specified operation of the oscillator and amplifier are summarized in Table II along with their measured performance. The figures for both the specification and the measured results are applicable over the temperature range -40 to $+85^{\circ} \mathrm{C}$ unless otherwise specified. A plot of the output power and frequency of the cascaded oscillator and amplifier as a function of ambient temperature is shown in Fig. 11.

The output power changes a little more than $1 \mathrm{~dB}$ over the entire temperature range, a virtue of the amplifier's operating in compression. The oscillator frequency can be varied by approximately $100 \mathrm{MHz}$ at $19 \mathrm{GHz}$, translating to $400-\mathrm{MHz}$ coverage in the 76-GHz band. The output power from the circuit is essentially flat as a function of frequency. This allows the source to 
TABLE II

COMPARISON OF THE MEASURED RESULTS FOR THE 19-GHz OSCILLATOR AND AMPLIFIER BUFFER STAGE WITH THE REQUIRED SPECIFICATION

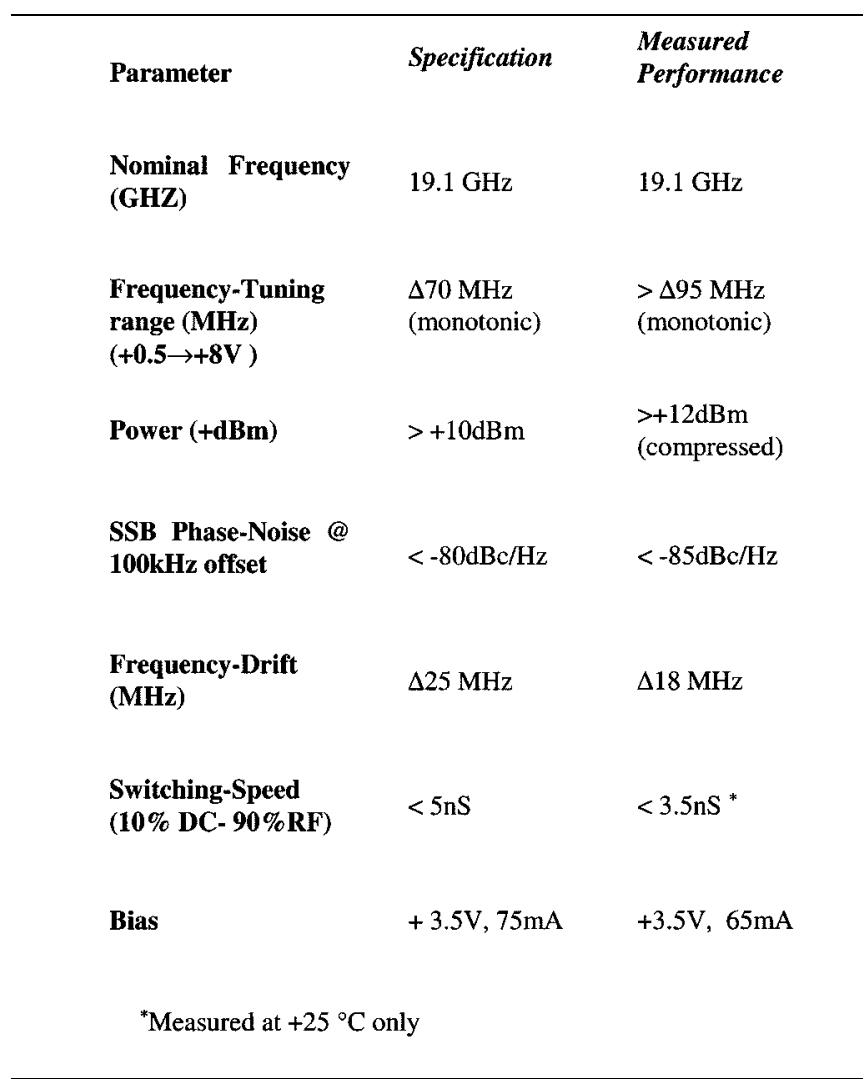

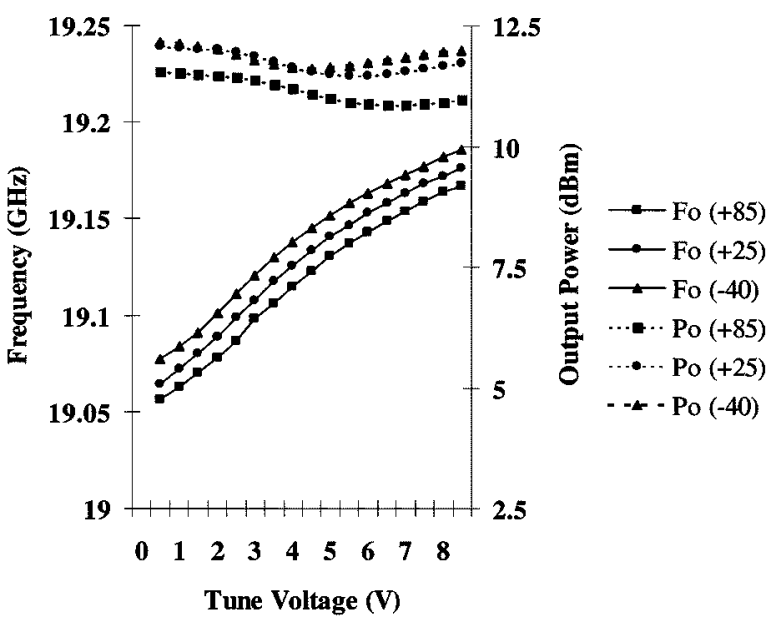

Fig. 11. Measured output power and frequency of the oscillator/amplifier as a function of temperature $\left(-40\right.$ to $\left.+85^{\circ} \mathrm{C}\right)$.

step between the transmit and receive frequencies determined by the IF bandwidth, plus have sufficient margin to cover sources of error such as temperature drift, frequency pulling, and aging, and still drive the passive doubler stage with near constant input power.

The switching speed of the $19-\mathrm{GHz}$ oscillator was measured using the conventional technique of splitting the oscillator output and driving a frequency discriminator. The output of the

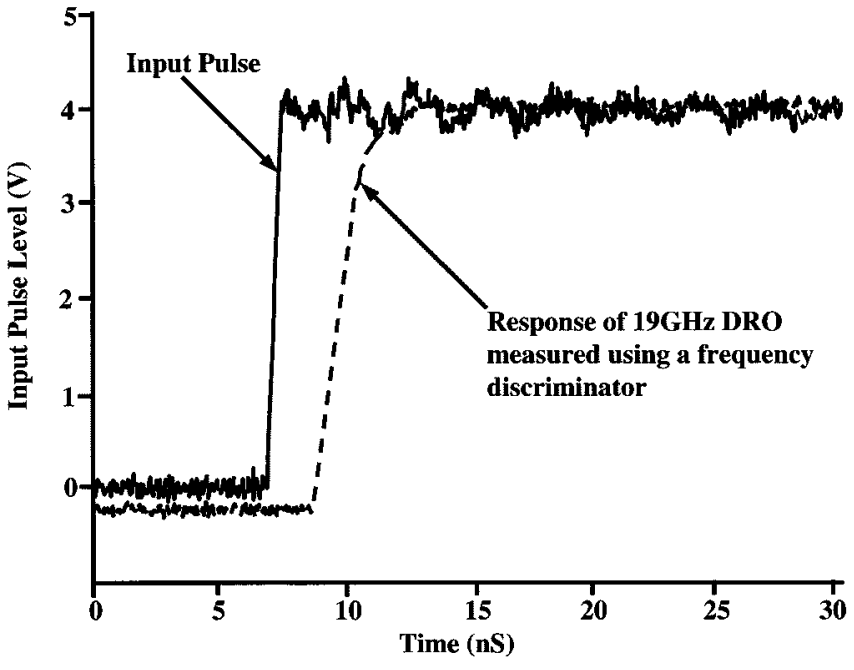

Fig. 12. Measured switching speed of the oscillator for a step change in the input tune-bias voltage.

discriminator is observed on an oscilloscope and compared with a reference trigger pulse from a pulse generator. The voltage step of the pulse generator is elected to represent the typical change in frequency required for the source to switch between the Tx and Rx (LO) frequency. The measured discriminator output is shown with the drive pulse in Fig. 12. The dc (10\%) to $\mathrm{RF}(90 \%)$ switching speed is $<4 \mathrm{nS}$.

2) 19-38 GHz Passive Frequency Doublers: The design of varactor-diode frequency doublers requires detailed and often complex synthesis methods owing to the sensitivity of the varactor diode to other circuit parameters [69]-[72] Although lossless reactive doublers have a theoretical efficiency of $100 \%$, the losses in the diode, the harmonic losses, and the losses in the matching circuits reduce the values achievable in practice. Table III summarizes the modeling techniques used for the electrical design of the frequency multiplier, although thermal considerations due to power dissipation in the diode junction are also important, as they restrict the maximum drive level of the doubler. These considerations arise in part due to the flip-chip diode attach but also due to the series circuit configuration and lack of direct thermal ground return.

In the series configuration, $\lambda_{g} / 4$ stubs at both the anode and cathode of the diode are used to separate the input and output networks of the frequency doubler. The shunt-stubs transform the doubler design into the design of input and output impedance matching circuits that match the impedance of the varactor diode at the input and output frequency, respectively. Table IV compares the specification design goals with measured results from the doubler. Note that the measured results with the exception of fundamental rejection were measured over the temperature range -40 to $+120^{\circ} \mathrm{C}$.

Information as to how the performance of the doubler will vary as a function of device parameters is required for assessing the selection of a die known to be good and, consequently, for yield for high-volume production. This was obtained for the passive doubler design by the generation of surface plots using conventional sensitivity analysis that illustrates, for example, the variance of the output power of the $19-38 \mathrm{GHz}$ doubler with 
TABLE III

SUMMARY OF THE 19-38 GHz DOUBLER CHARACTERISTICS AND SIMULATION INFORMATION

\begin{tabular}{|c|c|}
\hline \multirow{2}{*}{ Configuration } & $\begin{array}{l}\text { - Frequency doubler in series configuration using } \\
\text { microstrip. Diode is reverse biased }(+2.2 \mathrm{~V}) \text { to operate at } \\
\text { optimum efficiency point. }\end{array}$ \\
\hline & $\begin{array}{l}\text { - Diode is flip-chip mounted using conductive epoxy to } \\
\text { reduce parasitics associated with bond-wires }\end{array}$ \\
\hline \multirow{4}{*}{ Diode type } & - GaAs Abrupt-iunction varactor: $f=2700 \mathrm{GHz}$ \\
\hline & - $C_{j}(0)=0.15 \mathrm{pF}$ \\
\hline & - Barrier Potential $\phi=1.3 \mathrm{~V}$ \\
\hline & - Elastance-voltage exponent $\gamma=0.5$ \\
\hline Models & $\begin{array}{l}\text { - Diode model extracted from S-parameter and dc } \\
\text { measurements (junction) plus full-wave EM analysis of } \\
\text { the package }\end{array}$ \\
\hline \multirow{2}{*}{$\begin{array}{l}\text { Simulation } \\
\text { Techniques }\end{array}$} & $\begin{array}{l}\text { - Harmonic-balance analysis for nonlinear diode } \\
\text { operation }\end{array}$ \\
\hline & - Full-wave EM analysis of circuit discontinuities \\
\hline $\begin{array}{l}\text { Yield } \\
\text { Analysis }\end{array}$ & $\begin{array}{l}\text { - Surface plots and sensitivity analysis to determine } \\
\text { doubler operation as a function of diode parameters. } \\
\text { The parameters as then used to set diode specifications } \\
\text { so that assembly only uses known-good-die. }\end{array}$ \\
\hline
\end{tabular}

respect to the intrinsic diode parameters and package parasitic components. The surface plots not only indicate how well the design is centered with respect to variations in the important design variables but also extend to the selection or screening of appropriate diodes in the manufacturing process.

3) Millimeter-Wave Bond-Wire Interconnects: There is a common misconception that long bond-wire interconnects may not be used at millimeter-wave frequencies because of their high series inductance, resulting in poor return loss [73]-[75]. However, the alternative to bond-wire interconnections-flip-chip die attach-is not straightforward for large devices. While flip-chip attachment of discrete devices is relatively simple (so long as package parasitic components are properly accounted for), the flip-chip attachment of large MMICs and devices is fraught with material and mechanical attachment problems. It is extremely advantageous, therefore, because of both production and reliability considerations, to mount MMICs in a conventional manner and to use wire-bonds to interconnect the MMICs with the circuit substrate-assuming that a method that minimizes the bond-wire interconnect loss at millimeter-wave frequencies can be identified. One of the difficulties in accounting for the behavior of bond-wires is uncertainty of the equivalent circuit model at high frequencies [76], which varies with the number of bond-wires, bond-wire length, and diameter, plus the specific profile of the transition.

Fig. 13 shows a side view of the bond-wire interconnect between an MMIC chip and the GMIC substrate for this work. Automated ball-bonding machines used in high-volume manufacturing are capable of producing bond-wires of a particular pro-
TABLE IV

Comparison of the Design SPeCIFICATION With the MEasured RESULTS FOR THE 19-38-GHz DOUBLER

\begin{tabular}{lcc}
\hline Parameter & Specification & Measured Result \\
Frequency (GHz) & $19.12 \mathrm{GHz} \pm 100 \mathrm{MHz}$ & $19.125 \mathrm{GHz} \pm 125 \mathrm{MHz}$ \\
$\begin{array}{l}\text { Input Power } \\
(+\mathbf{d B m})\end{array}$ & $+10 \mathrm{dBm}<\mathrm{P}_{\circ}<+16 \mathrm{dBm}$ & $+10 \mathrm{dBm}<\mathrm{P}_{\circ}<+16 \mathrm{dBm}$ \\
$\begin{array}{l}\text { Conversion-Loss } \\
\text { (dB) }\end{array}$ & $<12 \mathrm{~dB}$ & $<9 \mathrm{~dB} \pm 0.5 \mathrm{~dB}$ \\
$\begin{array}{l}\text { Input/Output } \\
\text { Return Loss (dB) }\end{array}$ & $>15 \mathrm{~dB}$ & $>10 \mathrm{~dB}\left(\mathrm{P}_{\mathrm{o}}=10 \mathrm{dBm}\right)$ \\
& $>20 \mathrm{~dB}$ & $>15 \mathrm{~dB}$ \\
Rejection (dB) & & \\
& & \\
Input/Output & $50 \Omega$ & $50 \Omega$ \\
Impedance & & \\
\hline
\end{tabular}

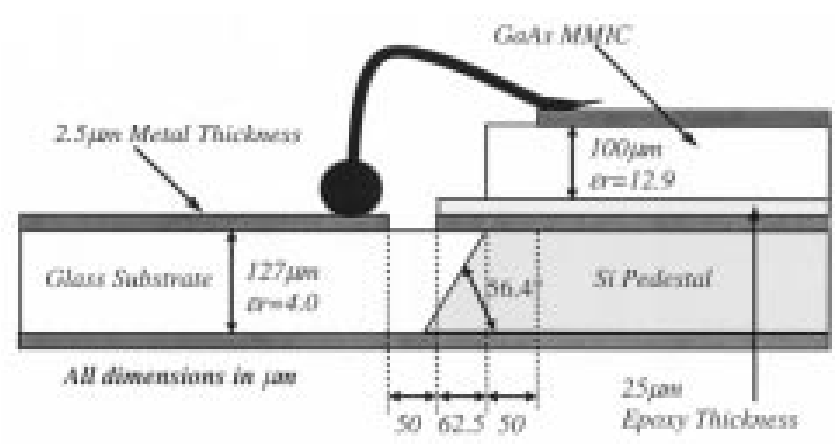

Fig. 13. Side profile of a $25-\mu \mathrm{m}$ ball wire-bond connection to an upright mounted MMIC. The dimensions reflect the typical wire-bond and MMIC mounting profile compatible with automated manufacturing equipment.

file both accurately and repeatedly. This is reassuring to the millimeter-wave circuit designer who, after designing a compensation network for a particular profile of bond-wire, can be confident that the tolerance on the dimensions of the bond-wire profile will be tightly held during manufacture. The caveat is that predictability is bought at the expense of a minimum bond-wire length, typically between 380 and $430 \mu \mathrm{m}$, which is long for millimeter-wave design. By treating bond-wire interconnects as a five-stage filter problem instead of as a single inductor that continually degrades RF performance [57], this problem can be solved.

The performance referenced in Table $\mathrm{V}$ is for a bond-wire length of $430 \mu \mathrm{m}$. This length is typical of a controlled bond-wire available with standard automated bonding machines. The interconnect referenced in [57] is specifically a glass-to-glass transition. Although a glass-GaAs interconnect has not been measured, similar performance may be expected, as the interconnect was designed to provide the same filter 
TABLE V

SUMMARY OF THE OPERATION OF BOND-WIRE INTERCONNECTS AT $77 \mathrm{GHz}$ WHEN USED AS PART OF A FILTER STRUCTURE

\section{Parameter}

Two- $25 \mu \mathrm{m}$ diameter ball bonds

Length $430 \mu m$
$S 11 @ 77 G H z$

Modeled

Performance

$22 \mathrm{~dB}$
$16 \mathrm{~dB}$

$S 11 @ 77 G H z$

Measured

Performance

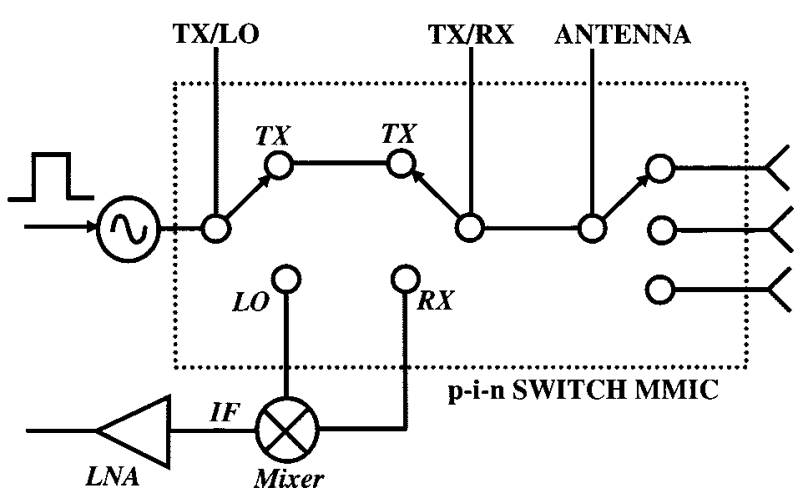

Fig. 14. Required functionality of the switch within the pulsed-Doppler radar.

response. This approach enables the use of upright wire-bonded MMICs for 76- $\mathrm{GHz}$ applications using existing production equipment and does not require costly development of flip-chip MMIC processes.

4) 76-GHz Reflective P-I-N Switch MMIC: Perhaps the essential requirement for a pulsed-Doppler radar system at 77 $\mathrm{GHz}$ is the ability to provide very fast switching-less than 5 $\mathrm{nS}$ - from transmit to receive functions to ensure good nearrange performance of the radar [56]. The MMIC switch also allows the antenna beams to be electronically steered, eliminating the need for multiple sources or mechanical antennas.

The GaAs p-i-n MMIC discussed in [56] was redesigned to improve switch isolation and insertion loss. Additional dc probe points are added to check the electrical continuity of the air bridges of each of the switch elements. Also, RF probe pads on each port allow each switch to be electrically screened to provide known good RF die. Fig. 14 illustrates the required functionality of the switch within the radar architecture.

The transmit pulse from the source is directed toward one of the three antenna ports. In transmit mode, the first two switch junctions are biased to direct the signal pulse (TX) toward the antenna. The transmit pulse is at a frequency $f_{1}$. After the pulse has been transmitted, the frequency of the source is changed to $f_{2}=f_{1}-I F$, where $I F$ represents the IF frequency of the heterodyne-recovered signal. The TX/LO switch is now set to the LO setting so that the source at $f_{2}$ acts as the LO drive for the signal recovery mixer. The TX/RX switch is changed to the RX setting so that the received signal is directed from the antenna and into the RX port of the mixer. The IF signal is then recovered and the information extracted. Information regarding

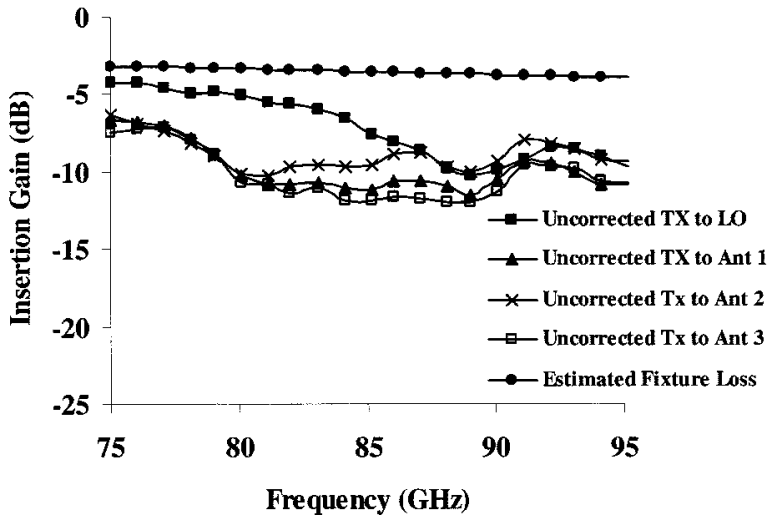

Fig. 15. Uncorrected insertion loss measurements for the Tx to antenna and Tx/LO paths of the switch. Also shown is the estimated fixture loss.

the switching speed of the p-i-n devices used in this MMIC may be found in [56].

A thru-reflect-line (TRL) calibration was used to extract the $S$-parameters of the diode junction using a single-diode switch as a test vehicle. This was performed with the diode biased in the reflective state (10-mA bias current) and in the transmission state $(0-\mathrm{V}$ bias voltage). On-wafer measurements of a singlepole/single-throw (SPST) switch demonstrated 0.8-dB insertion loss (including the transmission line) with an isolation of 26 $\mathrm{dB}$. The worst case isolation of the single-diode switch over the temperature interval -40 to $+105^{\circ} \mathrm{C}$ is $23 \mathrm{~dB}$ at $77 \mathrm{GHz}$. This performance is comparable to or better than other devices reported in the literature [77]-[80].

Full $S$-parameters were measured for the switch MMIC from 75 to $110 \mathrm{GHz}$. Fig. 15 displays the uncorrected insertion-loss values measured from 75 to $95 \mathrm{GHz}$. Since the frequency response of the microstrip-to-waveguide transition is not known, the values presented include any frequency response due to this transition and the fixture itself. The estimated insertion loss of the switch from this measurement is approximately $3 \pm 0.5 \mathrm{~dB}$ between the TX port and each of the three antennas at $76 \mathrm{GHz}$. The TX to LO path insertion loss is lower, as would be expected, and is approximately $1 \pm 0.5 \mathrm{~dB}$ at $76 \mathrm{GHz}$. The TX to antenna path contains three switches for a total insertion loss of $2.4 \mathrm{~dB}$. Adding in GaAs microstrip line losses on the MMIC brings the estimated loss very close to the measured values from the test fixture.

Fig. 16 displays the calibrated isolation values of each antenna under various bias states from 75 to $78 \mathrm{GHz}$. The switch provides isolation between the antenna ports of better than 23 $\mathrm{dB}$ at $76 \mathrm{GHz}$, regardless of the state of the switch. The final set of measurements used to characterize the switch is that of the LO-to-antenna isolation, shown in Fig. 17. The fixture measurements suggest that the LO-to-antenna isolation is approximately $19-24 \mathrm{~dB}$ for the MMIC.

5) 76-GHz Hybrid (Rat-Race) Mixer: The IF bandwidth of the pulsed-Doppler system represents an extremely small percentage of the operating frequency-about $0.25 \%$-indicating that the RF and $\mathrm{LO}$ frequencies are almost identical. A rat-race mixer is, therefore, an ideal choice for this application, as it provides RF-LO isolation. Additionally, it was found in modeling 


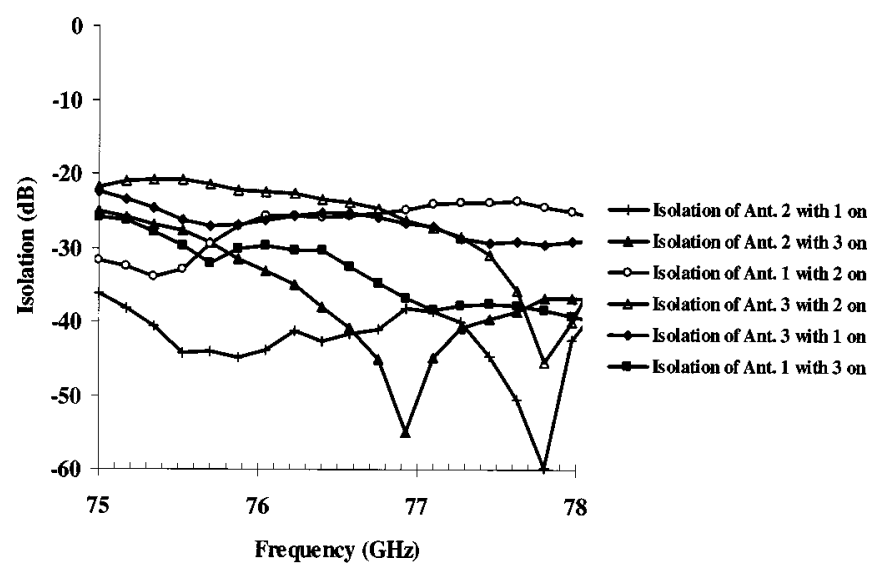

Fig. 16. Calibrated isolation between antenna ports under various conditions.

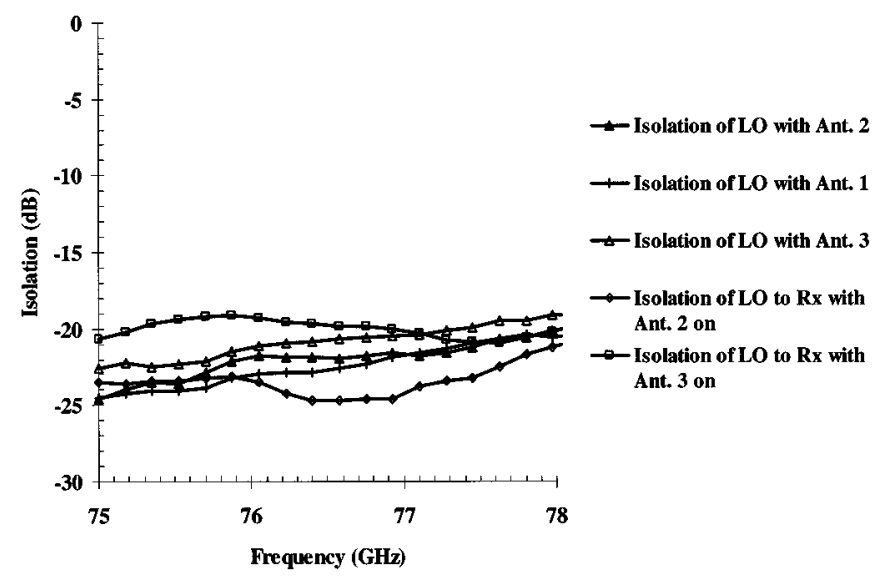

Fig. 17. LO isolation with respect to various states of the MMIC over the frequency range $75-78 \mathrm{GHz}$.

the design that matching circuits for the diodes were not required under the applicable bias. The mixer could be realized within a small area using two discrete Schottky diodes offering low-cost potential. Details of the diode model and the 76-GHz mixer design are described in [81]. Fig. 18 shows a photograph of the mixer test circuit used to measure the conversion loss as a function of LO input power. The Schottky diodes were flip-chip mounted and attached with conductive silver-loaded epoxy.

The measured conversion loss of the mixer was found to be $<6 \mathrm{~dB}$ for an $\mathrm{LO}$ drive level of +7 to $+14 \mathrm{dBm}$ [81]. This was

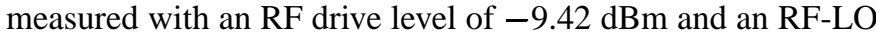
separation (IF bandwidth) equal to $180 \mathrm{MHz}$.

6) Microstrip-to-Waveguide Transition: A key element in maintaining a compact easy-to-manufacture low-cost package for this module is the realization of an MMIC-compatible microstrip-to-waveguide transition. Ideally, the transition should be easily integrated into the package, provide an efficient conversion from the low-impedance ( $50 \Omega$ ) microstrip environment to the higher impedance of waveguide, and be immune to typical dimensional and manufacturing tolerance variations. Another factor that may be considered desirable for harsh operating environments found in automotive applications is the ability for the transition to be made hermetic, thus rendering the circuitry feeding the waveguide port impermeable to moisture

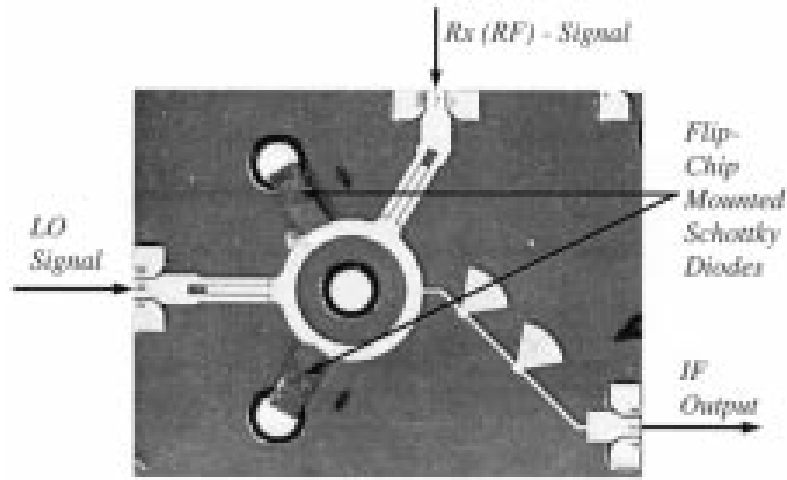

Fig. 18. The 76-77-GHz GMIC balanced mixer with flipped discrete Schottky diodes.

and other pollutants. In addition to the electrical requirements for the transition, there are several additional considerations pertaining to manufacturing and cost issues. The transition design is required to be insensitive to manufacturing tolerance for three reasons. First, alignment and tuning for specified performance by hand can be expensive, or at the very least time-consuming. Secondly, if the transition does not require tuning, the part count, and hence the cost, is usually reduced. Lastly, adding tuning compensation may render the manufacture and assembly of the part cumbersome and impractical for volume manufacturing.

As a result of the growing demand to find a low-cost transition between conventional planar circuitry and waveguide, the subject has attracted increasing interest. This has resulted in several techniques that have recently been reported in the literature [82]-[87], although none of the published techniques is wholly applicable for some of the design constraints of this specific application.

While keeping the constraints, requirements, and solutions in the literature in mind, we have developed a novel solution to this problem [60], [88].The transition that was conceived for this product development comprises a mode transformer to convert the transverse or, in the case of microstrip, quasi-transverse electric mode signal carried by the planar transmission line to a waveguide mode signal.

Fig. 19 illustrates the composition of the impedancematching structure within the transition. The $127-\mu \mathrm{m}$ glass substrate has full metallization on its reverse side with the exception of a patterned area immediately below the rectangular mode launch. This forms a window in the reverse side of the glass substrate that allows transmission of the electromagnetic wave into the waveguide impedance transformer. A photograph of the upper and lower sides of the glass substrate showing the three waveguide transitions and the three windows in the reverse side of the substrate is shown in Fig. 20. The lateral offset in distance between the three antenna launches on the upper side of the substrate allows for Si pedestals to be placed around each launch. The photograph of the reverse side of the substrate shows the etched glass "windows" in the ground plane of the substrate. A section through the glass substrate indicating how this window aligns with the MIM carrier is shown in Fig. 21. 


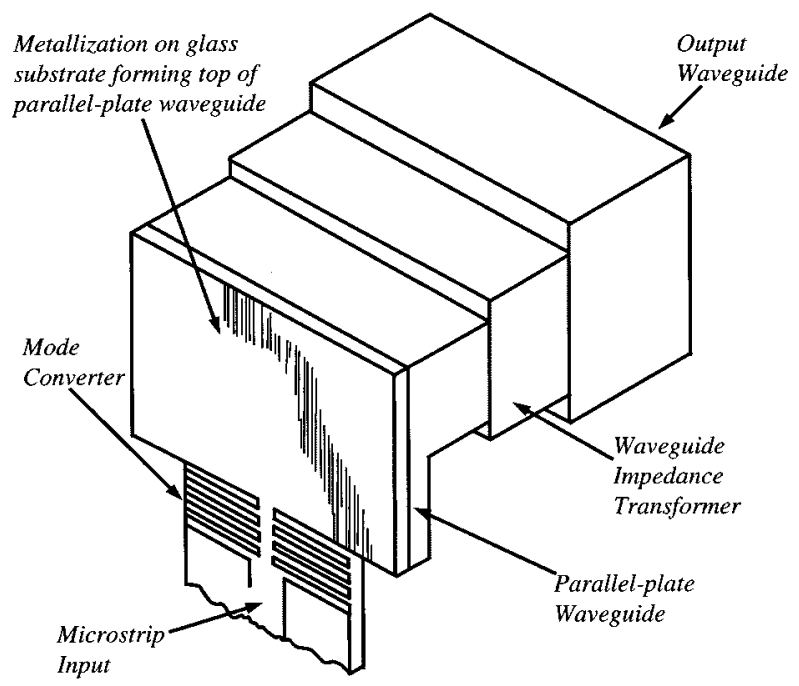

Fig. 19. Microstrip-to-waveguide transition using a mode converter and a waveguide impedance transformer.
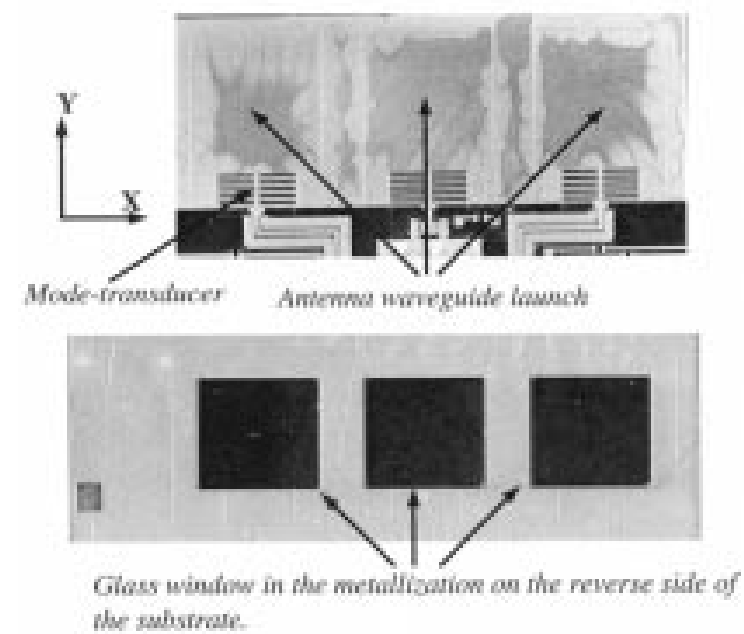

Fig. 20. Photograph of the three waveguide antenna launches from the front and rear sides of the glass substrate.

The mode converter or transducer-shown in detail in Fig. 22-transforms the quasi-transverse electromagnetic mode of the signal on the microstrip input transmission line into a parallel-plate waveguide mode in the glass substrate before continuing into several stepped impedance transformation sections of conventional rectangular waveguide. Because the carrier is metal, the walls that bound the dimensions of the first recessed portion are electrically conductive, thus forming the section of parallel-plate waveguide below the substrate. The combination of the mode transducer with several stages of impedance transformation as described results in a well-matched broadband transition. Furthermore, the dimensions and length of the impedance-matching sections are all compatible with the accuracy available from the MIM process used to manufacture the circuit carrier. As the carrier also forms the base of the module package, the stepped waveguide output transition is inexpensive to manufacture, and the transition between the planar transmission line and the waveguide output ports results in a low-cost millimeter-wave interface

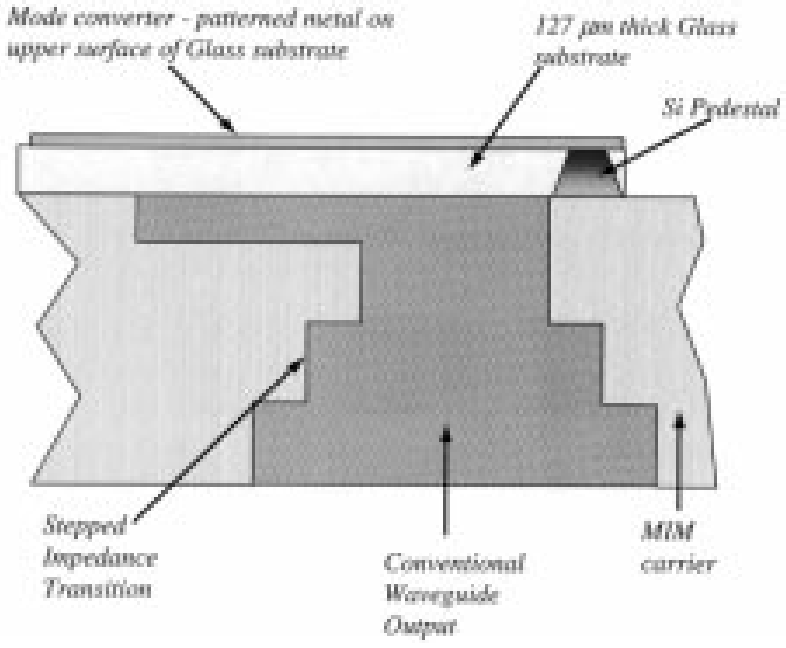

Fig. 21. Cross-section through the microstrip-to-waveguide transition illustrating the stepped impedance transition.

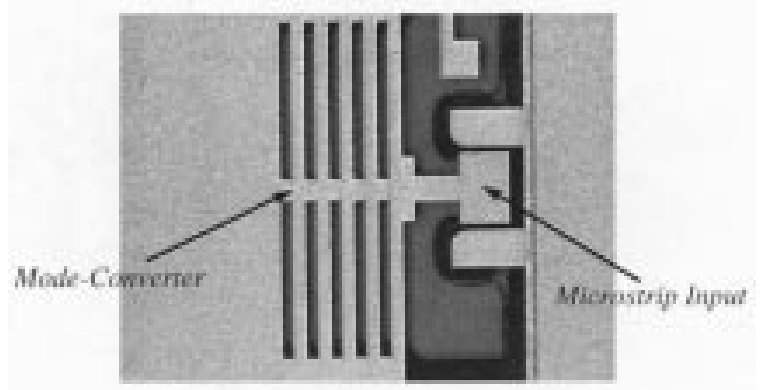

Fig. 22. Photograph of the glass-based mode converter used to transform the microstrip input to a waveguide mode in the substrate.

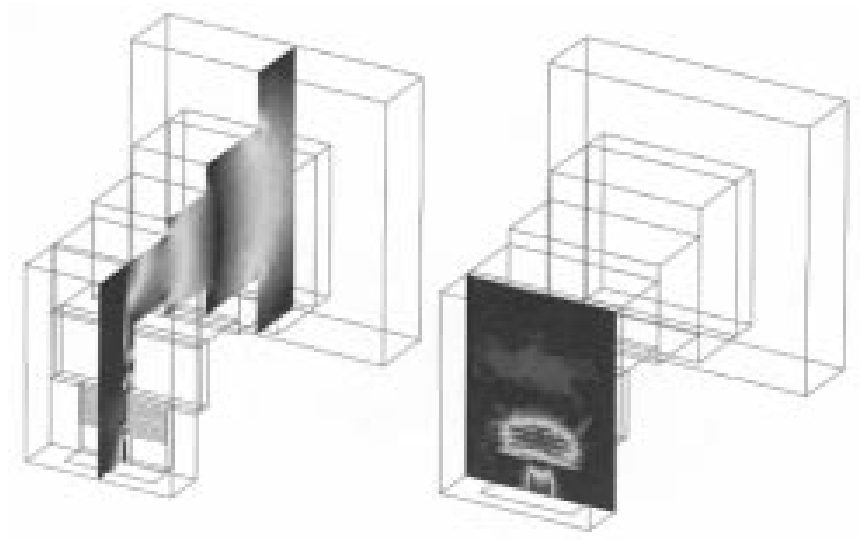

Fig. 23. Field structure for the mode transducer at the microstrip to waveguide interface and for the output waveguide as calculated using a finite-element analysis based field solver.

between the module and the antenna. The relative dimensions for the impedance matching sections were calculated using a finite-element analysis-based field solver in tandem with an optimization engine.

A plot of the calculated field structure for the mode transducer and the output waveguide is shown in Fig. 23. Varying the physical parameters within the simulation indicated that the operation of the transition would be relatively insensitive to typical variations in the dimensions of the MIM manufactured car- 


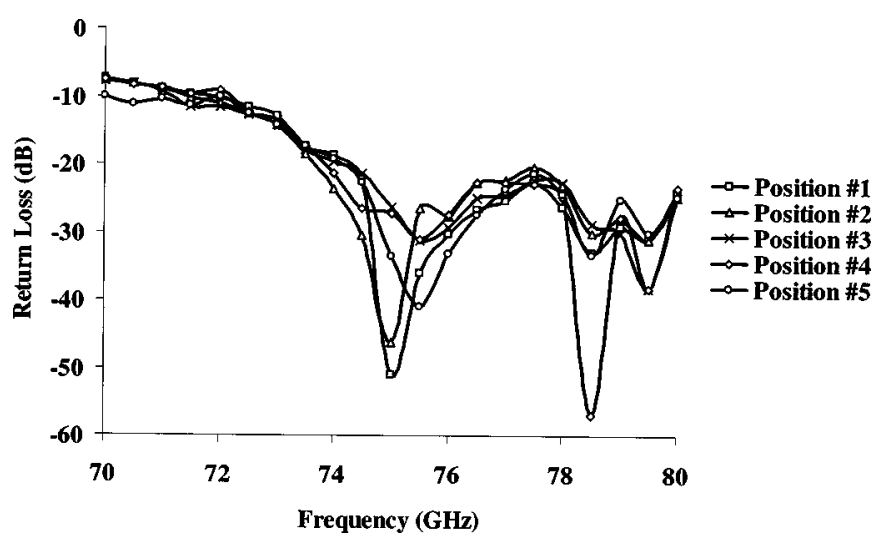

Fig. 24. Measured return loss of the microstrip-to-waveguide transition. The different responses are obtained as the registration between the substrate. The MIM carrier is varied by an offset of $\pm 100 \mu \mathrm{m}$ from the nominal placement position.

rier and stepped-impedance transition. In addition, photolithography ensures that the variation in the line width and spacing of the mode transducer will be small. The principal source of variation in the manufacturing process is, therefore, the accuracy of registration and alignment of the glass substrate on the MIM carrier. The effect of this misalignment is most critical in the lateral direction ( $x$-direction in Fig. 20). High-speed automated manufacturing equipment is capable of placing the substrate onto the carrier with an accuracy of better than $100 \mu \mathrm{m}$ (i.e., $\pm 50 \mu \mathrm{m}$ ) in both $y$ and $x$ directions.

To ascertain both the performance of the transition and its sensitivity to manufacturing tolerance, the return loss of the transition was measured in situ on the MIM carrier with the rectangular waveguide output terminated in RF absorber. The return loss of the transition was measured for substrates that had been misplaced by up to $200 \mu \mathrm{m}$ (i.e., $\pm 100 \mu \mathrm{m}$ ) in the $x$-direction. The increase in positional error was selected to allow for potential error in placement plus some postplacement variation in the substrate position due to effects such as epoxy creep. The results of these measurements are shown in Fig. 24. The transition return loss was measured at the microstrip input to the mode transducer using a CPW probe. The return loss of the transition may be seen to be better than $20 \mathrm{~dB}$ over the interval 74-80 GHz, even with a misalignment between the glass substrate and the recess in the carrier of up to $200 \mu \mathrm{m}$. The worst case return loss at $77 \mathrm{GHz}$ was measured to be better than $21 \mathrm{~dB}$. This performance represents a microstrip-to-waveguide transition that is better than many other complex transitions reported in the literature. Furthermore, the performance of the transition is robust and tolerant of typical manufacturing errors due to automated assembly equipment, and is thus ideal for low-cost millimeter-wave products.

\section{Measurement of The InTEgrated Module}

The $W$-band characteristics of the module are measured by integrating the module with an antenna. One of the design parameters of the module was to minimize the millimeter-wave interface length to reduce signal loss and mechanical complexity. This was achieved by designing the module to mount directly

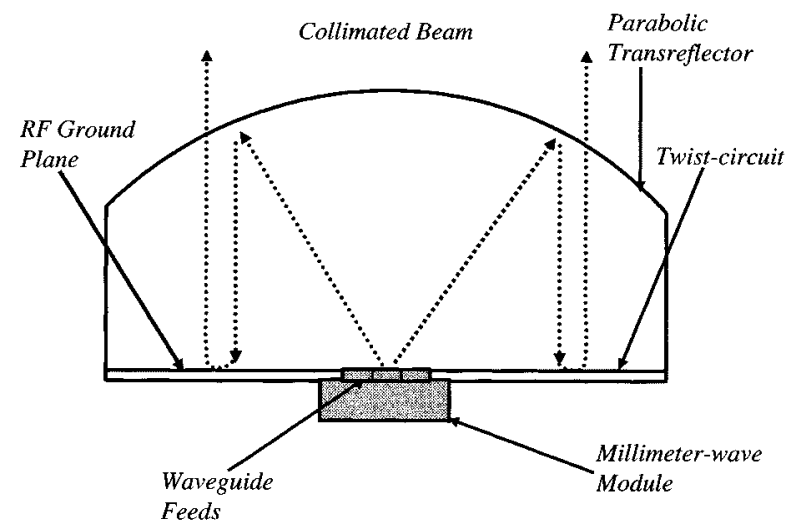

Fig. 25. Schematic diagram of the interface between the millimeter-wave module and the quasioptical antenna. The module is mounted on the reverse side of the antenna ground plane with the waveguide feeds forming a common RF ground.

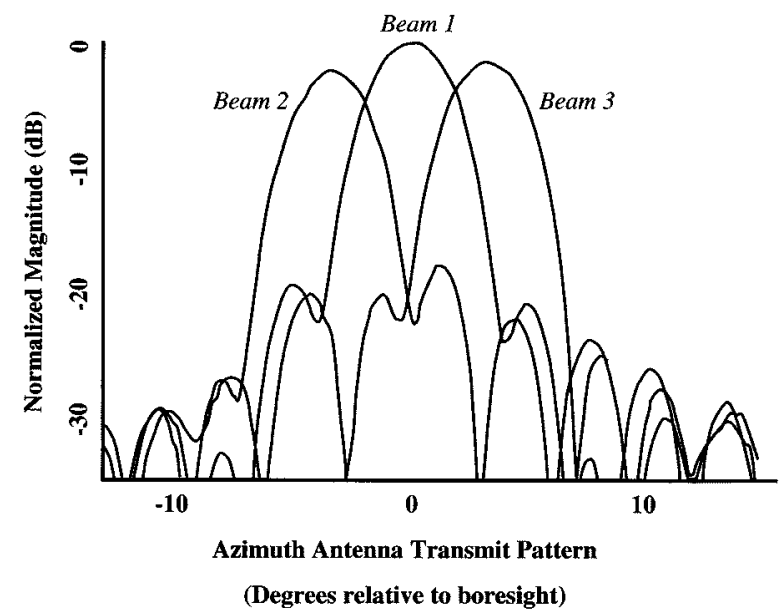

Fig. 26. Antenna transmit patterns for the module/antenna combination along the azimuth axis.

on the reverse side of an antenna ground plane and radiate directly into the input ports of the antenna. The antenna used for this demonstration was a quasi-optical lens-based design as currently used on commercially available ACC systems [89], [90]. The module waveguide ports illuminate a parabolic transreflector on the inner surface of the lens that either reflects or transmits the millimeter-wave signal depending upon the specific polarization. The reflected energy is then repolarized by a microstrip "twist-circuit" on the antenna ground plane to form a collimated beam; that is then transmitted. This process is shown schematically in Fig. 25. The antenna assembly and interface between the module and the antenna is shown in Fig. 3.

The printed circuit board (PCB) would ordinarily be used for the module drive circuitry plus the IF detection chain and DSP functions in an actual radar. However, for the purposes of testing the millimeter-wave functionality, the PCB contains only bias circuitry for the RF functions. The IF output from the module is connected to a surface-mount coaxial connector mounted on the PCB. It is not possible to do system-level measurements, therefore, but functions such as antenna patterns (TX and RX), LO leakage, and frequency pulling due to beam steering may be measured. 
TABLE VI

Summary of Some Measured Performance Parameters of the MiLLIMETER-WAVE MODULE
Parameter
Measured Performance
LO leakage
Minimum isolation between $\mathrm{Rx}$ and $\mathrm{Tx}>19.5 \mathrm{~dB}$

\section{Frequency} Pulling
Frequency pulling as the antenna beam is switched between channels $=2 \mathrm{MHz}$

Azimuth transmit patterns for the antenna are shown in Fig. 26 . The three beams are approximately $3^{\circ}$ wide with a $3^{\circ}$ separation. Sidelobe interference for each of the three beams may be seen to be $<15 \mathrm{dBc}$. Patterns for the azimuth receive function are substantially similar. These measured results are in accordance with expected results and are compatible with required system performance. Some additional measurements that confirm the correct operation of the module when integrated with an antenna are given in Table VI.

\section{SUMMARY}

A compact, complete transceiver module suitable for millimeter-wave $(77 \mathrm{GHz})$ vehicular radar applications has been described. All of the materials and assembly techniques used in the design are compatible with the low-cost objectives that the commercial millimeter-wave industry requires. At the component level, improved performance in millimeter-wave switch networks, and a new low-loss compact process- and assemblytolerant microstrip-to-waveguide transition have been shown. The measured results of each component have been presented, along with a discussion of their sensitivity to manufacturing variation. The operation of the module and the proof-of-concept of the integrated assembly technique have been demonstrated by the ability to measure antenna patterns that meet the system requirements.

It is appreciated that each application for millimeter-wave consumer systems will probably require very different levels of system operation. However, the issues of mechanical and electrical interfaces, compliance with standard high-volume manufacturing techniques, and pressures of aggressive pricing will be common enough that the principles and methodology used in designing this radar module will be relevant.

\section{ACKNOWLEDGMENT}

The authors are greatly indebted to the many individuals who contributed their time, advice, and encouragement during this development. Particular thanks are due to: D. Antopolsky, D. Curcio, K. Danehy, G. Davis, R. Descoteau, Dr. G. DiPiazza, S. Doyle, Dr. R. Egri, P. Ersland, F. Harrison, Dr. D. Hatzis, M. Hines, Dr. H.-R. Jen, Dr. J.-P. Lanteri, M. Leonard, P. Lessard, B. Lester, Dr. C. Licqurish, P. Onno, J. Putnam, D. Santesaunio, R. Sletten, E. Soshea, D. Swanson, D. Wells, Dr. Y.-H. Yun, and Dr. X. Zhang.

\section{REFERENCES}

[1] R. K. Jurgen, Ed., Object Detection, Collision Warning and Avoidance Systems: Society of Automotive Engineers, 1998.

[2] A. G. Stove, "Millimeter-wave radar for obstacle avoidance," in Proc. Military Microwaves, London, U.K., 1988, pp. 170-184.

[3] M. L. Skolnik, Introduction to Radar Systems, 2nd ed. New York: McGraw-Hill, 1980.

[4] W. Menzel, D. Pilz, and R. Leberer, "A 77GHz FMCW radar front-end with a low-profile low-loss printed antenna," in Proc. 1999 IEEE Int. Microwave Symp. (MTT-S), pp. 1485-1488.

[5] J. Mayock, C. M. Snowden, L. P. Ligthart, and P. Swart, "A wideband millimeter-wave front-end for automotive radar," in Proc. 1999 IEEE MTT-S, pp. 1501-1504.

[6] P. L. Lowbridge, "Low cost millimeter-wave radar systems for intelligent vehicle cruise control applications," Microwave J., pp. 20-33, Oct. 1995.

[7] L. H. Eriksson and S. Brodén, "High performance automotive radar," Microwave J., pp. 24-38, Oct. 1996.

[8] T. Heil et al., "Integrated radar receiver front ends," Microwave J., pp. 64-77, Mar. 1997.

[9] DELPHI Automotive Systems, "Forewarn collision warning system,", press release, 1999.

[10] Fujitsu Ten, "76 GHz millimeter-wave radar,", Press Release, 1999.

[11] N. Martin, "Adaptive cruise control hits the road," Auto. Ind., p. 113, Oct. 1998.

[12] Y. Hori, K. Onda, M. Funabashi, H. Mizutani, K. Maruhashi, A. Fujihara, K. Hosoya, T. Inoue, and M. Kuzuhara, "Manufacturable and reliable millimeter-wave HJFET MMIC technology using novel $0.15 \mu \mathrm{m}$ MoTiPtAu gates," in Int. Microwave Symp. Dig., 1995, pp. 431-434.

[13] P. M. Smith, "Status of InP HEMT technology for microwave receiver applications," in Int. Microwave Symp. Dig., 1995, pp. 1133-1136.

[14] M. Matloubian, "Advances in millimeter-wave FET MMIC technology," in Int. Microwave Symp. Dig., 1999, pp. 1743-1746.

[15] M. Camiade, D. Domnesque, P. Alleaume, A. Mallet, D. Pons, and H. Dambkes, "Full MMIC millimeter-wave front-end for a $76.5 \mathrm{GHz}$ adaptive cruise control car radar," in Proc. 1999 IEEE MTT-S, pp. 1489-1492.

[16] T. V. Kerssenbrock and P. Heide, "Novel 77 GHz flip-chip sensor modules for automotive radar applications," in Proc. 1999 IEEE MTT-S, pp. 289-292.

[17] J. R. Lamberg, M. J. Gawronski, J. J. Geddes, W. R. Carlyon, and R. A. Hart, "A compact, high performance $W$-band FMCW radar front-end based on MMIC technology," in Proc. 1999 IEEE MTT-S, pp. $1979-1800$

[18] A. Tessman et al., "A $77 \mathrm{GHz}$ GaAs pHEMT transceive MMIC for automotive sensor applications," in Proc. 1999 IEEE GaAs IC Symp., pp. 207-210.

[19] R. Isobe, C. Wong, A. Potter, L. Tran, M. Delaney, R. Rhodes, D. Jang, L. Nguyen, and M. Le, " $Q$ - and $V$-band MMIC chip set using $0.1 \mu \mathrm{m}$ millimeter-wave low noise InP HEMTs," in Int. Microwave Symp. Dig., 1995, pp. 1133-1136.

[20] H. Wang, K. W. Chang, D. Smith, G. S. Dow, K. L. Tan, A. K. Oki, and B. R. Allen, "A $W$-band source module using MMICs," IEEE Trans. Microwave Theory Tech., vol. 43, no. 15, pp. 1010-1016, May 1995.

[21] K. Sasaki, J. Utsu, K. Hoshino, T. Taguchi, and Y. Ueno, "InP MMIC's for $V$-band FMCW radar," in Proc. 1997 IEEE MTT-S, pp. 937-940.

[22] L. Verweyen, H. J. Siweris, M. Neumann, U. Schaper, R. Osorio, A. Werthof, S. Kudszus, H. Massier, H. Tischer, W. Reinert, A. Hulsmann, W. H. Haydl, T. Meier, W. Kellner, and M. Schlechtweg, "Coplanar transceiver MMIC for $77 \mathrm{GHz}$ automotive applications based on a nonlinear design approach," in Proc. 1998 RFIC Symp., pp. 33-36.

[23] T. Shimura, Y. Kawasaki, Y. Ohashi, K. Shirakawa, T. Hirose, S. Aoki, H. Someta, K. Makiyama, and S. Yokokawa, "76 GHz flip-chip MMIC's for automotive radars," in Proc. 1998 RFIC Symp., pp. 25-28.

[24] K. F. Lau, L. Liu, and S. Dow, "Recent MMW technology development and its military and commercial applications," in Proc. 1998 RFIC Symp., pp. 87-90.

[25] H. J. Siweris, A. Werthof, H. Tischer, U. Schaper, A. Schafer, L. Verweyen, T. Grave, G. Bock, M. Schlechtweg, and W. Kellner, "Low-cost GaAs pHEMT MMIC's for millimeter-wave sensor applications," IEEE Trans. Microwave Theory Tech., vol. 46, pp. 2560-2567, Dec. 1998.

[26] H. Kondoh et al., "77 GHz fully-MMIC automotive forward-looking radar," in Proc. 1999 IEEE GaAs IC Symp., pp. 211-214.

[27] N. Jain, "Designing commercially viable MM-wave modules," in Int. Microwave Symp. Dig., 2000, pp. 565-568. 
[28] H. Uchimura and T. Takenoshita, "A ceramic planar $77 \mathrm{GHz}$ antenna array," in Int. Microwave Symp. Dig., 1999, pp. 453-456.

[29] S. Zelubowski, "Low cost antenna alternatives for automotive radars," Microwave J., pp. 54-63, July 1994.

[30] K. Watanabe, T. Deguchi, and A. Nakagawa, " $V$-band planar gunn oscillators and VCO's on AlN substrates using flip-chip bonding technology," in Proc. 1999 IEEE MTT-S, pp. 13-16.

[31] A. L. Springer, C. G. Diskus, K. Lubke, and H. W. Thim, "A $60 \mathrm{GHz}$ MMIC-compatible TED-oscillator," IEEE Microwave Guided Wave Lett., vol. 5, pp. 114-116, Apr. 1995.

[32] H. Mizutani, N. Shida, T. Saryo, T. Kuwabara, T. Eda, T. Matsumura, and M. Funabashi, "76 GHz MMIC chipset for compact, low-cost and highly reliable automotive radar system," in Proc. 1999 IEEE RFIC Symp..

[33] T. Kashiwa, T. Ishida, T. Katoh, H. Kurusu, H. Hoshi, and Y. Mitsui, " $V$-band high-power low phase-noise monolithic oscillators and investigation of low phase-noise performance at high drain bias," IEEE Trans. Microwave Theory Tech., vol. 46, pp. 1559-1565, Oct. 1998.

[34] M. J. Vaughan, R. C. Compton, S. Duncan, D. Tu, and S. Weinreb, "Monolithic $W$-band voltage controlled oscillator," in Int. Microwave Symp. Dig., 1996, pp. 337-340.

[35] A. Bangert, M. Schlechtweg, M. Lang, W. Haydl, W. Bronner, T. Fink K. Kohler, and B. Raynor, " $W$-band MMIC VCO with a large tuning range using a pseudomorphic HFET," in Int. Microwave Symp. Dig., 1996, pp. 525-527.

[36] Y. Kawasaki, K. Shirakawa, Y. Ohashi, and T. Saito, "60 GHz monolithic oscillator using InGaP/InGaAs/GaAs HEMT technology," in Int Microwave Symp. Dig., 1995, pp. 541-544.

[37] D. C. W. Lo, K. W. Chang, R. Lin, E. W. Lin, H. Wang, M. Biedenbender, G. S. Dow, and B. R. Allen, "A single-chip $W$-band transceiver with front-end switching receiver for FMCW radar applications," in Int. Microwave Symp. Dig., 1995, pp. 873-876.

[38] H. Wang, K. W. Chang, D. C. W. Lo, L. T. Tran, J. C. Cowles, T. R. Block, G. S. Dow, A. Oki, D. C. Streit, and B. R. Allen, "A $62 \mathrm{GHz}$ monolithic InP-based HBT VCO," IEEE Microwave Guided Wave Lett., vol. 5, pp. 388-390, Nov. 1995.

[39] U. Guttich, H. Shin, U. Erben, C. Gaessler, and H. Leier, "24-27 GHz dielectrically stabilized oscillators with excellent phase-noise properties utilizing InP/InGaAs HBTs," in Proc. 1999 IEEE MTT-S, pp. 729-732.

[40] M. S. Heins, T. Juneja, J. A. Fendrich, J. Mu, D. Scott, Q. Yang, M. Hattendorf, G. E. Stillman, and M. Feng, " $W$-band InGaP/GaAs HBT MMIC frequency sources," in Int. Microwave Symp. Dig., 1999, pp. 239-242.

[41] J. R. Pelliccio, "Predict component effects on MTI radar performance," Microwaves and RF, pp. 57-64, June 1996.

[42] K. Hosoya, T. Inoue, M. Funabashi, and K. Ohata, "Systematic evaluation and analysis for $60 \mathrm{GHz}$ dielectric resonators coupled to a microstrip line on a GaAs substrate," IEEE Trans. Microwave Theory Tech., vol. 46 , pp. 352-358, Apr. 1998.

[43] T. V. Kerssonbrock and P. Heide, "Novel 77 GHz flip-chip sensor modules for automotive radar applications," in Int. Microwave Symp. Dig., 1999, pp. 289-292.

[44] D. D. Li, S. C. Luo, C. Pero, X. Wu, and R. M. Knox, "Millimeter-wave FMCW/monopulse radar front-end for automotive applications," in Int. Microwave Symp. Dig., 1999, pp. 277-280.

[45] H. J. Siweris, H. Tischer, T. Grave, and W. Kellner, "A monolithic $W$-band HEMT VCO with feedback topology," in Int. Microwave Symp. Dig., 1999, pp. 17-20.

[46] M. Funabashi, T. Inoue, K. Ohata, K. Maruhashi, K. Hosoya, M Kuzuhara, K. Kanekawa, and Y. Kobayashi, "A $60 \mathrm{GHz}$ MMIC stabilized frequency source composed of a $30 \mathrm{GHz}$ DRO and doubler," in Int. Microwave Symp. Dig., 1995, pp. 71-74.

[47] R. Hess, "MMIC's for commercial applications: The low-cost high volume production techniques," in Proc. Microwave Millimeter-Wave Monolithic Circuits Symp., 1998, pp. 3-6.

[48] G. Bechtel, "The 1999 outlook for GaAs IC markets and technology," in Proc. IEEE GaAs IC Symp., 1999, pp. 7-9.

[49] N. P. Morenc, "MMIC's for automotive radar application," in Int. Microwave Symp. Dig., 1996, pp. 39-41.

[50] L. Raffaelli, "Millimeter-wave automotive radars and related technology," in Int. Microwave Symp. Dig., 1996, pp. 35-38.

[51] L. Tran, R. Isobe, M. Delaney, R. Rhodes, D. Jang, J. Brown, L. Nguyen, M. Le, M. Thompson, and T. Liu, "High performance, high yield millimeter-wave MMIC LNA's using InP HEMTs," in Int. Microwave Symp. Dig., 1996, pp. 133-136.

[52] A. Werthof, T. Grave, and W. Kellner, " $W$-band amplifier fabricated by optical stepper lithography," in Int. Microwave Symp. Dig., 1999, pp. 689-692.
[53] N. P. Morenc, "MMIC's for automotive radar applications," in Int. Microwave Symp. Dig., 1996, pp. 39-41.

[54] K. Takahashi, S. Fujita, T. Yoshida, H. Sakai, and M. Sagawa, "An advanced millimeter-wave flip-chip IC integrating different kinds of active devices," in Int. Microwave Symp. Dig., 1996, pp. 1619-1622.

[55] B. A. Ziegner, R. J. Sletten, N. Jain, and S. R. Brown, "Millimeter-wave module with an interconnect from and interior cavity," U.S. Patent 6064286.

[56] J. Putnam, M. Barter, K. Wood, and J. LeBlanc, "A monolithic GaAs PIN switch network for a $77 \mathrm{GHz}$ automotive collision warning radar," in Proc. 1997 RFIC Symp., pp. 225-228.

[57] T. P. Budka, "Wide bandwidth millimeter-wave bond-wire interconnects," IEEE Trans. Microwave Theory Tech., to be published.

[58] P. Chinoy, N. Jain, P. Li, J. Goodrich, and C. Souchons, "Manufacture of low-loss microwave circuits using HMIC technology," in Proc. 1994 IEEE MTT-S, pp. 1137-1140.

[59] R. Perko, et al., "MMIC vs. hybrid: Glass microwave IC's rewrite the rules," Microwave J., no. 11, pp. 67-78, 1988.

[60] N. Jain, "Transverse electric or quasitransverse electric mode to waveguide mode transformer," U.S. Patent 6087907.

[61] N. Popovic, "Review of some types of varactor tuned DRO's," Applied Microwave and Wireless, pp. 62-70, August 1999.

[62] E. C. Niehenke and P. A. Green, "A low-noise $L$-band dielectric resonator stabilized microstrip oscillator," in Proc. 1987 IEEE MTT-S, pp. 193-196.

[63] N. Popovic, "TDRO with a varactor-diode in the gate-circuit," in Proc. XLI ETRAN Conf., vol. II, June 1997.

[64] R. K. Mongia, "On the accuracy of approximate methods for analyzing cylindrical dielectric resonators," Microwave J., pp. 146-150, Oct. 1991.

[65] T. Itoh, "New method for computing the resonant frequencies of dielectric resonators," IEEE Trans. Microwave Theory Tech., vol. MTT-25, pp. 52-54, Jan. 1977

[66] D. Kajfez, "Variational improvement of the Itoh and Rudokas model," in Dielectric Resonators. Reading, MA: Artech House, 1986.

[67] K. A. Michalski, "Rigorous analysis methods," in Dielectric Resonators. Reading, MA: Artech House, 1986.

[68] P. Guillon, "Coupling parameters between a dielectric resonator and a microstripline," IEEE Trans. Microwave Theory Tech., vol. MTT-33, Mar. 1985.

[69] C. B. Burckhardt, "Analysis of varactor frequency multipliers for arbitrary capacitance variation and drive," Bell Syst. Tech. J., pp. 675-693, 1965.

[70] S. A. Maas, Nonlinear Microwave Circuits. Reading, MA: Artech House, 1988

[71] P. Penfield Jr. and R. P. Rafuse, Varactor Applications. Cambridge, MA: MIT Press, 1962.

[72] T. C. Ho, S. W. Chen, K. Pande, and P. D. Rice, "A $W$-band integrated power module using MMIC MESFET power amplifiers and varactor doublers," IEEE Trans. Microwave Theory Tech., vol. 46, pp. 2388-2294, Dec. 1993.

[73] T. Krems, W. Haydl, H. Massler, and J. Rudiger, "Millimeter-wave performance of chip interconnections using wire-bonding and flip-chip," in Proc. 1996 IEEE MTT-S, pp. 247-250.

[74] G. Hanke and W. Nohr, "A new chip interconnection technique for ultra high-speed and millimeter-wave applications," in Proc. 1996 IEEE MTT-S, pp. 1611-1614.

[75] T. Hirose, K. Makiyama, K. Ono, T. M. Shimura, S. Aoki, Y. Ohashi, S. Yokokawa, and Y. Watanabe, "A flip-chip MMIC design with CPW technology in the $W$-band," in Proc. 1998 IEEE MTT-S, pp. 2276-2282.

[76] H. Y. Lee, "Wideband characterization of a typical bonding wire for microwave and millimeter-wave integrated circuits," IEEE Trans. Microwave Theory Tech., vol. 43, pp. 63-68, Jan. 1995.

[77] A. Klaassen and J. M. Dieudonne, "77 GHz monolithic MMIC Schottkyand PIN-diode switches based on MESFET and silicon-SIMMWIC technology," in Int. Microwave Symp. Dig., 1995, pp. 1631-1634.

[78] H. Takasu, F. Sasaki, H. Kawasaki, H. Tokuda, and S. Kamihashi, "W-band SPST transistor switches," IEEE Microwave Guided Wave Lett., vol. 6, pp. 315-316, Sept. 1996.

[79] M. Case, M. Matloubian, H. Sun, D. Choudhury, and C. Ngo, "Highperformance $W$-band GaAs PIN diode single-pole triple-throw switch CPW MMIC," in Int. Microwave Symp. Dig., 1997, pp. 1047-1051.

[80] E. Alekseev, "W-band InGaAs/InP PIN diode monolithic integrated switches," in GaAs IC Symp. Dig., 1995, pp. 253-256.

[81] T. P. Budka, D. Antopolsky, and M. Pizzella, "A $77 \mathrm{GHz}$ mixer for automotive radar," Microwave J., pp. 132-134, Jan. 2000.

[82] W. Menzel and A. Klaassen, "On the transition from ridged waveguide to microstrip," in Proc. 19th Eur. Microwave Conf., 1989, pp. 1265-1269. 
[83] W. Grabherr, B. Huder, and W. Menzel, "Microstrip to waveguide transition compatible with mm-wave integrated circuits," IEEE Trans. Microwave Theory Tech., vol. 42, pp. 1842-1843, Sept. 1994.

[84] M. Davidovitz, "Wide-band waveguide to microstrip transition and power divider," IEEE Microwave Guided Wave Lett., vol. 6, pp. 13-16, Jan. 1996.

[85] L. Hyvonen and A. Hujanen, "A compact MMIC-compatible microstrip to waveguide transition," in Proc. 1996 IEEE MTT-S, pp. 875-878.

[86] W. Simon, M. Werthen, and I. Wolff, "A novel coplanar transmission line to rectangular waveguide," in Proc. 1998 IEEE MTT-S, pp. 257-260.

[87] F. J. Villegas, D. I. Stones, and H. A. Hung, "A novel waveguide-tomicrostrip transition for millimeter-wave module applications," IEEE Trans. Microwave Theory Tech., vol. 47, pp. 48-54, Jan. 1994.

[88] A. Alexanian, N. Jain, and T. Budka, "Planar transmission line to waveguide transition for a microwave signal," U.S. Patent 6087907 , July 2000.

[89] G. R. Huguenin, "Compact microwave and millimeter-wave radar," U.S. Patent 5455 589, Oct. 1995.

[90] G. R. Huguenin et al., "Compact microwave and millimeter-wave radar," U.S. Patent 5680 139, Oct. 1997.

I. Gresham (S'90-M'93) received the B.Eng. and Ph.D. degrees in electronic engineering from the University of Leeds, U.K., in 1988 and 1994, respectively.

He was with Marconi Radar and Control Systems, Leicester, U.K. until 1990, when he joined the Microwave and Solid-State Group at the University of Leeds. His doctoral work was on low-noise oscillators. He joined M/A-COM, U.K., in 1993, where he worked on microwave signal generation techniques and MMIC design for communications applications. He joined the Corporate Research and Development Group, Lowell, MA, as a Principal Engineer in July 1998. Since that time, he has been involved in the design and development of millimeter-wave radar and communications systems for commercial markets.

Dr. Gresham is a Member of the IEEE Microwave Theory and Techniques Society and the IEEE Electron Devices Society.

N. Jain (S'90-M'91) received the B.Tech. degree in electronics from the Indian Institute of Technology, Madras, in 1986 and the M.S. and Ph.D. degrees in solid-state electronics from Rensselaer Polytechnic Institute, Troy, NY, in 1989 and 1991, respectively.

His Ph.D. work focused on MESFET, pHEMT, and diode-gate MESFET control devices. From 1991 to 1998, he was with Corporate R\&D, M/A-COM, Lowell, MA, as a Principal and then a Senior Principal Engineer. His work focused on the commercial use of EM simulators, passive and active circuit modeling, multilayer PCB modeling, high-power PIN switches, millimeter-wave circuits, and millimeter-wave module development. He has worked on various commercial millimeter-wave projects, including the design and production of an LMDS receiver at $28 \mathrm{GHz}$ and the design of a $24-\mathrm{GHz}$ collision avoidance radar. He was also the Technical Lead of a team that designed a single substrate radar module for a 77-GHz ACC application. Since 1998, he has been with the Electrical and Computer Engineering Department, Indian Institute of Science, Bangalore, as an Assistant Professor. His current research interests include millimeter-wave circuits and millimeter-wave communication modules, noise, and passive circuit losses. He has more than 20 publications in international conferences and journals and has received five U.S. patents.

Prof. Jain received the IEEE Graduate Student Fellowship Award in 1990.

T. Budka (S'92-M'95) received the A.B. degree in physics from Dartmouth College, Hanover, NH, in 1989, the M.A. degree in physics from The University of Rochester, Rochester, NY, in 1991, and the Ph.D. degree in electrical engineering from the Department of Electrical Engineering and Computer Science, The University of Michigan, Ann Arbor, in 1995.

His dissertation was titled "Microwave Circuit Electric Field Imaging Systems." From 1995 to 1998, he was an MMIC Designer with Texas Instruments/Raytheon TI Systems developing flip-chip embedded transmission line MMIC power amplifiers. From 1998 to 1999, he was with M/A-COM's Research and Development group designing/developing a low-cost $76-\mathrm{GHz}$ radar system on a single chip. He has received two patents and has numerous publications in refereed journals. Presently, he is a Staff RF Design Engineer with RF Micro Devices' Boston Design Center focusing on advanced product development. ciety.
A. Alexanian (S'92-M'93) received the B.S. degree (summa cum laude) from Virginia Polytechnic Institute and State University, Blacksburg, in 1991, the M.S. degree from the University of Michigan at Ann Arbor in 1993, and the $\mathrm{Ph} . \mathrm{D}$. degree from the University of California at Santa Barbara in 1997, all in electrical engineering.

His thesis work comprised electromagnetic analysis of periodic structures using the finite-difference time-domain technique and development of broadband spatial power-combining schemes. From 1997 until 1999, he was with the M/A-COM Corporate Research and Development Group. His work involved 77-GHz automotive radar sensors and included design and development in the areas of antennas, circuits, and packaging. Since 1999, he has been with the RFMD Boston Design Center. He is currently designing RFICs for the Digital Cellular product line.

N. Kinayman (S'94-M'96) was born in Ankara, Turkey, in 1968. He received the B.Sc. and M.Sc. degrees from the Middle East Technical University, Ankara, in 1990 and 1993, respectively, and the Ph.D. degree from Bilkent University, Ankara, in 1997, all in electrical engineering.

He is a Principal Engineer in M/A-COM's Corporate Research and Development department, Lowell, MA. He was a Test and Design Engineer for Aselsan, a company that specializes in military electronics. His main responsibility was to design computer-controlled and manual radio test stations. His main professional interest is the numerical solution of electromagnetic problems and model extraction of passive microwave printed circuits. He has also written full-wave electromagnetic simulation software using the method of moments to simulate microstrip circuits.

B. Ziegner completed his studies at the University of Arizona, Tucson, and Arizona State University, Tempe.

He has been with M/A-COM since 1982 and is currently a Senior Principal Engineer of M/A-COM's Corporate R\&D organization. His responsibilities include material evaluation, electrical-mechanical design, and project management. He is also responsible for technology development for advanced microwave packaging focusing on low-cost manufacturing methods applicable to both commercial and defense applications. He has worked on GaAs MESFETs, wafer-level testing/modeling, radar T/R module packaging, glass-silicon microwave integration, and, most recently, the development of packaging technology for millimeter-wave automotive sensors. Prior to joining M/A-COM, he was with Motorola, Phoenix and Scottsdale, AZ, for 20 years. He has received seven patents with others pending and has published six papers covering various aspects of device and circuit packaging. He , and

Mr. Ziegner is an affiliate of IEEE Components, Packaging, and Manufacturing Technology Soceity and IMAPS.

S. Brown, photograph and biography not available at the time of publication.

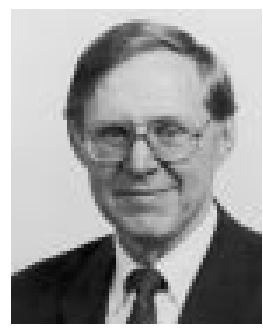

P. Staecker (S'63-M'72-SM'87-F'95) received the B.S. degree from the Massachusetts Institute of Technology (MIT), Cambridge, and the Ph.D. degree from the Polytechnic Institute of Brooklyn, Brooklyn, NY, both in electrical engineering.

While with the MIT Lincoln Laboratory, Cambridge, he designed microwave devices and circuits, and developed measurement techniques for their application to satellite communications. As Director of Engineering in the Corporate Research and Development Center, he helped facilitate the company's transition from government to commercial focus. In 1998, he retired from AMP M/A-COM as Director of Research and Development. He serves on the Editorial Advisory Review Boards on Microwave Journal, Applied Microwave and Wireless Magazine, and Artech House Publishers, Norwood, MA.

Dr. Staecker has served for many years in technical and administration capacities in the IEEE Microwave Theory and Techniques Society (IEEE MTT-S). He was general chair of the 1991 IEEE MTT-S International Microwave Symposium (IMS) and was technical chair of the 2000 IEEE MTT-S IMS. He served as president of the IEEE MTT-S in 1993 and is current its awards chair. He is the Division IV director-elect for the year 2000. In addition to his IEEE activities, he has chaired the Technical Working Group on RF Components, National Electronics Manufacturing Initiative from 1996 to 1999, and is a member of the National Research Council NIST-EEEL Assessment Panel. 